\title{
Three-dimensional Deep-tissue Functional and Molecular Imaging by Integrated Photoacoustic, Ultrasound, and Angiographic Tomography (PAUSAT)
}

Mucong Li

Duke University

Nathan Beaumont

SonoVol. Inc. https://orcid.org/0000-0002-1135-5785

Chenshuo Ma

Duke University

Juan Rojas

SonoVol, Inc.

Tri Vu

University at Buffalo, State University of New York

Max Harlacher

SonoVol. Inc.

Graeme O'Connell

SonoVol. Inc.

Ryan Gessner

SonoVol. Inc.

Hailey Kilian

University of Buffalo

Ludmila Kasatkina

Albert Einstein College of Medicine https://orcid.org/0000-0003-3935-5389

Yong Chen

Duke University

Qiang Huang

Duke University

\section{Xiling Shen}

Duke University https://orcid.org/0000-0002-4978-3531

Jonathan Lovell

University at Buffalo, State University of New York https://orcid.org/0000-0002-9052-884X

\section{Vladislav Verkhusha}

Albert Einstein College of Medicine 


\section{Tomek Czernuszewicz}

SonoVol. Inc.

Junjie Yao ( $\nabla$ junjie.yao@duke.edu )

Duke University https://orcid.org/0000-0002-2381-706X

\section{Article}

Keywords: deep-tissue imaging, photoacoustic imaging, ultrasound imaging, acoustic angiography, functional imaging, molecular imaging, photoswitchable protein

Posted Date: December 16th, 2021

DOI: https://doi.org/10.21203/rs.3.rs-1143622/v1

License: (c) (i) This work is licensed under a Creative Commons Attribution 4.0 International License. Read Full License

Version of Record: A version of this preprint was published at IEEE Transactions on Medical Imaging on January 1st, 2022. See the published version at https://doi.org/10.1109/TMI.2022.3168859. 


\section{Three-dimensional Deep-tissue Functional and Molecular} Imaging by Integrated Photoacoustic, Ultrasound, and Angiographic Tomography (PAUSAT)

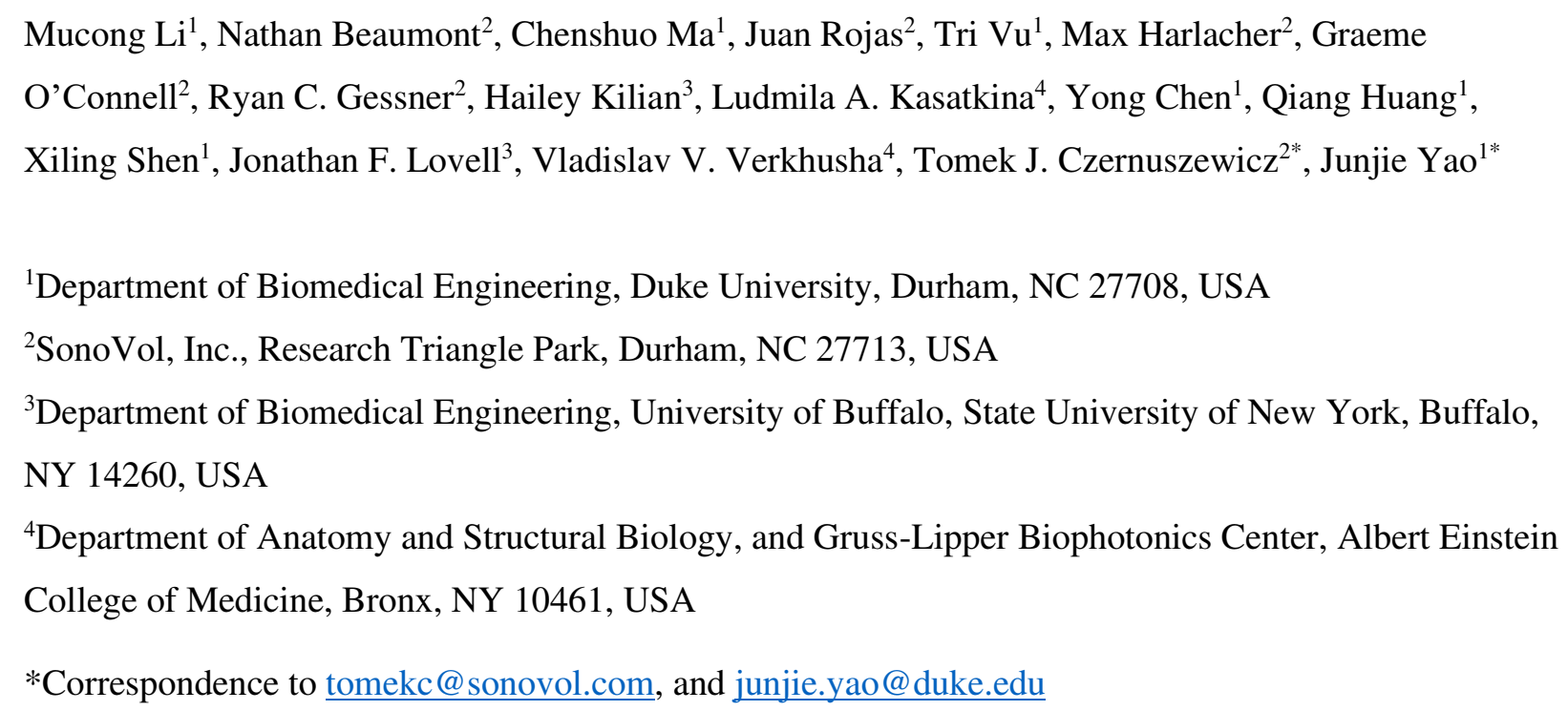

Abstract: Non-invasive small-animal imaging technologies, such as optical imaging, magnetic resonance imaging and $\mathrm{x}$-ray computed tomography, have enabled researchers to study normal biological phenomena or disease progression in their native conditions. However, existing smallanimal imaging technologies often lack either the penetration capability for interrogating deep tissues (e.g., optical microscopy), or the functional and molecular sensitivity for tracking specific activities (e.g., magnetic resonance imaging). To achieve functional and molecular imaging in deep tissues, we have developed an integrated photoacoustic, ultrasound and angiographic tomography (PAUSAT) system by seamlessly combining light and ultrasound in a non-invasive manner. PAUSAT can perform three imaging functions simultaneously with complementary contrast: high-frequency B-mode ultrasound imaging of tissue morphology, microbubbleenabled acoustic angiography of vasculature, and multi-spectral photoacoustic imaging of molecular probes. PAUSAT can provide three-dimensional (3D) multi-contrast images that are automatically co-registered, with high spatial resolutions at large depth. Using PAUSAT, we conducted proof-of-concept in vivo experiments on various small animal models: monitoring longitudinal development of placenta and embryo during mouse pregnancy, tracking biodistribution and metabolism of near-infrared organic dye on the whole-body scale, and 
detecting genetically-encoded breast tumor expressing photoswitchable phytochromes. These results have collectively demonstrated that PAUSAT has broad applicability in biomedical research, providing comprehensive structural, functional, and molecular imaging of small animal models.

Keywords - deep-tissue imaging, photoacoustic imaging, ultrasound imaging, acoustic angiography, functional imaging, molecular imaging, photoswitchable protein

\section{Introduction}

Non-invasive small-animal imaging technologies have evolved rapidly over the past decades with increasingly broad applications, enabling researchers to study the progression of biological events, evaluate the progression of various diseases, and monitor responses to treatment $[1,2]$. All major medical imaging techniques such as x-ray computed tomography (CT), positron emission tomography (PET) and magnetic resonance imaging (MRI), have been adapted for in vivo small-animal imaging, usually with smaller formfactors and improved spatial resolutions compared with their clinical counterparts [3-8]. Based on different contrasts, small-animal imaging modalities can provide complementary information of the biological tissues. For example, small-animal PET/CT maps tissue's morphology and metabolic activities with deep penetration, but both PET and CT employ ionizing radiation, in addition to the poor soft tissue contrast in CT and complicated workflow in PET, which hinders longitudinal studies [9-12]. Small-animal MRI uses non-ionizing radiofrequency waves to map soft tissue information with excellent contrast and spatial resolution but suffers from a low imaging speed and high cost that hamper some in vivo applications and limit throughput [1, 13]. To overcome the above limitations, there need to be new imaging modalities that can provide non-ionizing functional and molecular imaging in deep tissues.

While light is usually non-ionizing and intrinsically sensitive to tissue's functional and molecular contrast, high-resolution optical imaging technologies such as multi-photon microscopy often lack the penetration depth for deep tissue studies [14, 15]. Bioluminescence and fluorescence imaging have also been widely applied for monitoring tumor development with high sensitivity and contrast, but they suffer from poor spatial resolution at depths [16, 17]. Ultrasound tomography (UST), on the other hand, can provide deep tissue information with high spatial and temporal resolutions, but so far still lacks molecular sensitivity [18-23]. Seamlessly 
combining the rich optical absorption contrast and the deep ultrasound penetration, photoacoustic tomography (PAT) is the promising optical imaging modality that can achieve functional and molecular imaging at large depths with high spatial resolutions [24-26]. In PAT, the energy of incident photons is absorbed by tissue chromophores and is partially or completely converted into ultrasonic waves via non-radiative relaxation. The ultrasonic waves are subsequently detected to reconstruct tomographic images of the original optical energy deposition in tissue [27, 28]. Because ultrasound waves are only weakly scattered in soft tissues, PAT can achieve high spatial resolution at depths larger than $10 \mathrm{~mm}$, far beyond the optical diffusion limit. Moreover, due to optical absorption contrast, PAT has intrinsic functional and molecular sensitivity, which is highly desired for preclinical research on small animal models. We have previously demonstrated deep-tissue photoacoustic imaging of cancer metastasis with high sensitivity, using near-infrared photoswitchable phytochromes [29]. Nevertheless, due to the lack of endogenous contrast in the extravascular space, PAT typically cannot provide morphological information of the soft tissue. Moreover, due to partial acoustic detection coverage and/or sparse spatial sampling, PAT often suffers from streaking artifacts and missing structures.

High-performance small animal imaging needs high spatial resolution, high temporal resolution, deep penetration, high structural fidelity, high throughput, and functional and molecular sensitivity, all in the same system. None of the above imaging technologies can meet this need alone, given their respective shortcomings. Therefore, it is most logical to integrate multiple imaging modalities with complementary contrast mechanisms, compatible system configurations, and comparable length scales. In this work, we report a technical advance in small animal imaging: the integrated photoacoustic, ultrasound and angiographic tomography (PAUSAT) platform. As a single imaging system, PAUSAT can provide comprehensive information about organ structures via ultrasound imaging (US), vascular morphology via acoustic angiography (AA), and molecular composition via photoacoustic imaging (PA) [30, 31]. All three imaging modes were engineered to function in the same system with shared scanning mechanisms, synchronized controlling, and parallel signal detection. More importantly, the three imaging modes share the same field of view and the images are automatically co-registered. We have thoroughly characterized the performance of PAUSAT, and demonstrated its non-invasive structural, functional, and molecular imaging with sub-millimeter resolutions at depths beyond $10 \mathrm{~mm}$. PAUSAT has enabled multiple comprehensive small animal studies that are not feasible 
by individual imaging modes, including longitudinal monitoring of placenta and embryo development during mouse pregnancy, continuous tracking of biodistribution and metabolism of a second-window near-infrared (1000 nm-1700 nm) organic dye on the whole-body scale, and highly-sensitive detection of breast cancer expressing genetically-encoded photoswitchable phytochrome. All these results have collectively demonstrated that PAUSAT is a powerful imaging tool and may find a broad range of applications for basic research.

\section{Results}

Configuration of the PAUSAT system. The schematic and working principle of the PAUSAT system is shown in Figs. 1a-b. PAUSAT was implemented from a modified Vega robotic imaging system (SonoVol, Inc., Durham, NC), which employs two ultrasound transducers to achieve the three imaging modes. The first transducer is a customized dual-element focused wobbler, with a high-frequency inner element $(35 \mathrm{MHz})$ and a low-frequency outer element (2 $\mathrm{MHz}$ ). The two elements are concentrically aligned with a shared focal length. The second transducer is a 128-elememnt linear array (5 MHz) (L7-4, Philips, USA). The dual-element focused transducer is used for US and AA imaging, providing high-resolution images of mouse organ structures and vasculature perfusion at deep regions of interest. The US mode uses the inner element for both high-frequency signal transmission and receiving. The AA mode uses the outer element to burst microbubbles flowing in blood vessels and uses the inner element to receive super-harmonic bubble bursting signals, resulting in high-contrast vasculature images [32]. The linear array, coupled with a dual-branch optical fiber bundle flanking its lateral sides, is used for the PA imaging to achieve high-speed molecular imaging with large penetration depth. The excitation light source for the PA imaging is a pulsed Nd: YAG laser (Q-smart 850, Quantel Laser, USA; wavelength: $1064 \mathrm{~nm}$; pulse reptation rate: $10 \mathrm{~Hz}$ ).

A FPGA based control box is programmed to synchronize the laser firing, motor stage motion, and PA/US data acquisition. The US and AA signals are acquired by a single-channel high-speed DAQ card. The PA signals are acquired by a programmable ultrasound scanner (Vantage 256, Verasonics, USA). While the linear array provides axial cross-sectional images for the PA mode with back-projection-based image reconstruction, the fast-scanning of the wobbler provides axial cross-sectional images for the US and AA mode (Supplementary Video 1). The two transducers are mounted on a robotically actuated carriage with their relative positions fixed. The carriage moves along a two-dimensional motorized stage for acquiring 3D 
images with a large field of view [32]. The key acoustic, optical (except the laser), scanning, and electronical components are fully immersed in a hydrocarbon fluid filled reservoir, which improves the system's lubrication, acoustic coupling, and heat dissipation. The reservoir has an optically and acoustically transparent imaging window on the top. The animal lies on the top of the imaging window with a natural prone position, which can reduce the respiration-induced motion artifacts.

Characterization of PAUSAT. We characterized the PAUSAT system's spatial resolutions as shown in Figs. 1c-d. The cross-section of a carbon fiber ( $\sim \mu \mathrm{m}$ in diameter), representing a line target, was imaged by the wobbler with the US mode and by the linear array with the PA mode. The lateral resolution and axial resolution of the PA mode at $25 \mathrm{~mm}$ from the linear array surface were $412.5 \mu \mathrm{m}$ and $418.7 \mu \mathrm{m}$, respectively. The US mode had an axial resolution of $118.7 \mu \mathrm{m}$ and a lateral resolution of $206.9 \mu \mathrm{m}$, which are close to the theoretical values. The spatial resolutions of the AA mode should be slightly worse than the US mode, since the same wobbler is used but the AA mode only involves one-way ultrasound focusing. The wobbler can also work at different frequencies, resulting in different spatial resolutions and penetration depths, due to the frequency-dependent attenuation of ultrasound waves. As shown in Fig. 1e, cross-sectional US images of a pregnant mouse were acquired at three transmission frequencies of $15 \mathrm{MHz}, 25$ $\mathrm{MHz}$ and $35 \mathrm{MHz}$. High-frequency US images provide better spatial resolution but smaller penetration depth.

Validation of the PAUSAT system on phantoms. The three imaging modes in PAUSAT provide complementary information of the same target. We first validated the imaging performance of PAUSAT by imaging a knot phantom made of a soft tube (inner diameter, 200 $\mu \mathrm{m}$; outer diameter $300 \mu \mathrm{m}$ ) to mimic a blood vessel. The tube was perfused with water, microbubbles, or an NIR-II dye BIBDAH for the US, AA and PA mode, respectively. All three imaging modes were able to delineate the shape of the knot based on different contrasts, as shown in Fig. 2a. The US image provides a sharp image of the tube wall, however, also shows the imaging window membrane which the tube is resting on. AA mode displays similar resolution; however, it exhibits almost no background signal and, consequently, high contrast of the tube lumen. The PA mode has worse resolution than the US and AA mode, due to the weak elevational focusing of the linear array and lower ultrasonic center frequency. The crosssectional images are shown in Fig. 2b, with the axial and lateral profiles shown in Fig. 2c. In the 
axial direction, the US image shows both top and bottom walls of the tube, while the PA and AA images show the lumen of the tube.

Although the PA mode can be used for vascular imaging with hemoglobin as the endogenous contrast [33-35], the AA mode can provide vascular images with better contrast and resolution. On the other hand, the PA mode has the unique molecular sensitivity with a variety of exogenous contrast agents [36-38], which cannot be provided by the US or AA mode. Here we tested the molecular imaging of the PA mode using an NIR-II BIBDAH dye that has strong optical absorption at $1064 \mathrm{~nm}$. Figure 2d shows the PA images of BIBDAH-filled tubes with different concentrations. The quantitative results in Fig. 2e show that the PA signal amplitude is proportional to the dye concentration, which is the foundation for spectroscopic PA measurement. The PA signals of whole bovine blood were also acquired at $1064 \mathrm{~nm}$, which were comparable to that of the BIBDAH dye with a $50 \mu \mathrm{g} / \mathrm{mL}$ concentration. The strong PA performance of BIBDAH dye is consistent with the optical absorption spectra of the BIBDAH dye and blood in Fig. 2f [39].

PAUSAT validation on small animals. To further validate the system for in vivo small animal studies, a mouse model was developed by subcutaneously implanting an artificial Matrigel "tumor" mixed with the BIBDAH dye (no tumor cells included) close to the liver cavity as shown in Fig. 3a, mimicking the targeted exogenous PA contrast agents tagging tumor cells [40]. The PA, US, and AA modes were then used sequentially to scan the artificial tumor region. Figure $\mathbf{3 b}$ shows the maximum amplitude projection images along the elevational direction. The artificial tumor was clearly imaged by the US mode as a hypoechogenic mass with a contrast to noise ratio (CNR) of 3.01, since the Matrigel had low acoustic reflectivity [41]. The AA mode provided abundant vascular network around the tumor implant but was not able to clearly delineate the tumor region. The PA image highlighted the strongest BIBDAH signals from the tumor region with a CNR of 14.38 , much better than the US image. The complementary nature of the three imaging modes can be more clearly visualized from the coronal cross-sectional images shown in Fig. 3c. The liver region was located by the US image, and the AA image provided the fine vascular architecture of the liver. The PA image highlighted the tumor implant. Figure 3d shows the 3D rendering of the merged images combining the three imaging modalities (Supplementary Video 2). Figure 3e further shows the superimposed images of the US, AA 
and PA modes, illustrating the relative positions of the liver, the hepatic blood vasculature and the artificial tumor.

Longitudinal imaging of the mouse pregnancy. Non-invasive imaging of the developing embryo in vivo with normal pregnant conditions is highly desired for developmental biology and reproductive science. Traditional optical imaging cannot clearly visualize the deep-seated embryos without implanting intravital windows which are invasive and may lead to embryo death [42]. PAUSAT was then used for longitudinal non-invasive imaging of in vivo mouse pregnancy to solve this issue. In this experiment, the developing embryos and placenta were imaged by the US mode (Fig. 4a) and the PA mode (Fig. 4d) every two days from E9 to E17, and by the AA mode (Fig. 4e) at E9, E13 and E17. The development of a representative embryo over the 9 days is shown in the close-up US images in Fig. 4b and Supplementary Video 3. The embryonic body part such as the head, the spinal cord and the tail were clearly resolved. The crown-rump length (CRL), which is the length of the embryo from the top of its head to the bottom torso, is an important clinical parameter to estimate the embryo's gestation age (GA) [43]. We measured the CRL values of the visible embryos at each imaged gestation age from the US images (Fig. 4c). A linear fitting was performed to show the growth rate of the embryos. To verify the accuracy of the CRL measurements, we adapted a CRL prediction model describing the change of the CRL with GA as $C R L=-9.42+1.09(G A)+0.0281(G A)^{2}$ [43]. From both our US measurements and predictions model, it can be observed that CRL increased approximately linearly with the gestation age. Figure 4d and Supplementary Video 4 show the PA images of the mother mouse at the abdominal area. The green dotted lines indicate the same landmark blood vessels at different time points. The same embryo can then be located from the PA images based on the landmark vessels as indicated by the green rectangles. In the AA images in Fig. 4e and Supplementary Video 5, we can clearly visualize blood vessels in the maternal organs, as well as the placentas since E13. The embryos were not visible in the AA mode because the microbubbles could not pass the placental barrier [44]. The three-dimensional AA images are shown in Supplementary Video 6. In addition, since the placental size is an indicator of fetal development and pregnancy outcome, we measured placental diameter (PD) and placental thickness (PT) in the US and AA images for all the embryos at E11, E13 and E15, where the placentas were well identified. The measured placental sizes at these gestation age agreed with 
the predicting models as $P D=-11.96+2.09(G A)-0.046(G A)^{2}-0.0005(G A)^{3}$ and $P T=4.10-$ $1.14(G A)+0.115(G A)^{2}-0.0031(G A)^{3}[43]$.

Tracking the biodistribution of an NIR-II dye. It is critically important to monitor the biodistribution and metabolism of organic chemicals at the whole-body level for drug development and treatment evaluation. PAUSAT is fully capable of molecular imaging with high detection sensitivity. As a proof of concept, we demonstrated the application of PAUSAT for monitoring the metabolism of a BIBDAH dye in a mouse. The coronal PA images of the mouse before and after administrating BIBDAH via the tail vein are shown in Fig. 5a. A clear increase in the PA signal strength was observed in the major blood vessels right after the dye injection. The PA signal of the dye at $0.1 \mathrm{mg} / \mathrm{mL}$ was $\sim 5$ times higher than that of the blood. The blood vessels imaged by the PA mode were mostly detected along the elevational direction, while much fewer vessels were detected along the lateral direction, since PA signals generated from blood vessels are strongly orientation dependent [45]. The PA signals from the lateral blood vessels were not detected by the full aperture of the linear transducer array, which resulted in their low visibility. Within 4 hours, the PA signals increased by 5 times in the liver, indicating that the dye was accumulated in the liver, consistent with previous results [36]. Figure 5b shows representative cross-sectional images of the liver region acquired at three time points. The images are shown in a logarithmic scale to highlight deeper signals. These results show that the penetration depth of the PA mode was improved after injecting the dye, as marked by the white dotted circle. Figure 5c shows the PA signal dynamics from the major blood vessels in the skin and from the liver. The 3D superimposed PA and US image of the liver region is shown in Fig. 5d. The PA signals in the liver continuously increased over four hours, showing the accumulation of dye in the liver. By contrast, the PA signals in the skin blood vessels reached a plateau one hour after injection. The persistent dye signals in the blood vessels are due to the relatively high concentration of dye. The slow clearance of the dye from the blood stream to the liver did not affect the PA signals in the blood vessels.

Detecting reversibly-photoswitching tumors. In addition to exogenous organic dyes, endogenous genetic reporters, such as reversibly photoswitchable phytochromes, have become increasingly popular in PA molecular imaging due to their high biocompatibility and much enhanced detection sensitivity. One of these phytochrome proteins is Bphpl that originates from rhodopseudomonas [29, 46]. From the optical absorption spectra in Fig. 6a, Bphpl exhibits two 
switchable absorbing states: the Pfr state and the Pr state. The Pfr state is the ground state or ON state. With 730-790 nm optical illumination, BphPl undergoes photoconversion to its Pr state or OFF state, at which the optical absorption is shifted to 630-690 $\mathrm{nm}$. The molar extinction coefficient of Bphpl at the ON state (at $750 \mathrm{~nm}$ ) is $\sim 70$ times higher than that of oxygenated hemoglobin [29]. This photoswitching is reversible between the two states. In this experiment, we implanted transgenic Bphpl-expressing 4T1 tumor cells into the mouse flanks and imaged the tumor regions after three weeks with US, AA, and PA modes. For PA imaging, we used a 750-nm pulsed laser to both generate PA signals and switch off Bphpl, and a 635-nm CW laser to switch the proteins back on (Fig. 6b). Axial-view US and AA images are shown in Fig. 6c, with the two tumor locations identified. The tumors showed low echogenicity in the US image, and no noticeable increase in blood perfusion was observed inside the tumors from the AA image. The PA images of the tumors acquired with BphPl at the ON and OFF states were dominated by the background signals from hemoglobin (Fig. 6d). By contrast, the differential PA image between the ON and OFF state can clearly detect the tumor signals with the background hemoglobin signals effectively suppressed (Fig. 6e). The differential PA image was then compared with the US image (Fig. 6f), and the Bphpl-expressing tumor locations correspond well with the US-detected tumor positions. Representative PA signals from the tumor and background over several switching cycles are shown in Fig. 6g, in which repeatable photoswitching was observed from the tumors but not from the blood.

\section{Discussion}

In this work, we have presented a 3D non-invasive small-animal imaging system that combines photoacoustic, ultrasound, and acoustic angiography imaging in a single device. PAUSAT can provide complementary information about biological tissues: structural information with US mode, vascular information with AA mode, and molecular information with PA mode. We performed in vitro studies to characterize the system and showed that high spatial resolutions are achieved by all three imaging modes for deep tissues. We also demonstrated the system's in vivo performance in various proof-of-concept small animal studies, including longitudinal monitoring of mouse pregnancy, tracking the biodistribution of an NIR-II dye, and detecting reversible photoswitching tumors. All the results collectively show the flexibility and 
versatility of the PAUSAT system for small animal studies, and potential wide applicability in a variety of basic research.

Compared with the US and AA mode that use high-frequency ultrasound, the PA mode has worse lateral and axial resolutions, which can be further improved by using an ultrasound array with a higher central frequency and broader bandwidth. The elevational resolution of the PA mode is limited by the elevational focusing, which can be improved by changing the scanning geometry or applying advanced 3D reconstruction algorithms [47-49]. The penetration depth of the US and AA mode is scalable, depending on the transmission ultrasound frequency. The penetration depth of the PA mode is fundamentally limited by the optical attenuation of the tissue, and can be improved by illuminating the mouse from multiple angles [50].

Another limitation of the current PAUSAT system is that two ultrasound transducers are used for three imaging modes that have increased the complexity in the image co-registration and prevented acquiring all three images simultaneously. While the US and AA modes share the same ultrasound transducer and therefore are naturally registered, the PA mode needs to be coregistered with the US and AA mode using geometric information of the two transducers. Geometric registration works well given stationary of the imaging subject, however if the animal moves between scans (e.g., muscle spasm, incomplete anesthesia) registration fidelity may decrease. Another method to register images from different modalities is matching the blood vessels from both AA and PA images with non-rigid registration techniques [51]. Artificial markers attached to the animal that can be imaged by all three imaging modes could also be used to achieve more precise image registration.

The stock concentration of microbubble used in the AA mode is $2 \times 10^{9}$ bubbles $/ \mathrm{ml}$. The relatively high microbubble concentration is necessary due to the passive bubble clearance in the blood stream and the active bubble bursting by the ultrasound pulses. Previous studies have shown that without active bursting, the lifetime of microbubbles in the blood stream depends on the bubble size. Microbubbles with a diameter of $1 \mu \mathrm{m}$ can last for $\sim 5 \mathrm{mins}$ in the blood circulation before the bubble signals drop to the background level $[52,53]$. In our study, we used a size-sorting process to produce larger microbubbles with a diameter of $5 \mu \mathrm{m}$ that could last longer in the circulation system and thus allow a longer imaging time window [54]. Nevertheless, the AA mode actively burst the bubbles to generate high-frequency harmonic signals, which accelerates the bubble clearance rate. Also, in our current setting, the AA mode averages 
multiple repeated frames at each location in order to improve the contrast of the major blood vessels, which, can suppress the signals from small vessels, especially capillaries with low flow speed. This multi-frame averaging further speeds up the microbubble clearance. Ultimately, we have an average microbubble lifetime of $~ 20$ mins in the blood circulation, which is sufficient to cover a field of view of $20 \mathrm{~mm}$ by $40 \mathrm{~mm}$. Reducing the frame averaging can prolong the bubble lifetime at the price of image contrast. An alternative approach to maintain a longer bubble circulation time is to use a continuous infusion pump by placing a catheter into the vein.

In the pregnant mouse imaging (Fig. 4), the major organs of the mouse embryos can be well resolved with the US mode and the maternal blood vasculature can be seen with the AA mode. While microbubbles are considered safe for in vivo imaging, the active bubble bursting may be a safety concern $[55,56]$. The bubble busting may generate high-pressure micro-streams that can change the permeability of the syncytiotrophoblast, which is the maternal-embryo exchange interface and a protective barrier [44, 57]. Previous studies on the blood-brain barrier have shown that this bubble-induced effect on the barrier permeability is transient, if present [58]. In our experiments, we did not observe any adverse effects on the maternal and embryo functions over the gestation period after the start of imaging. The US images confirmed the normal progress of pregnancy up to the delivery. We also noticed that the embryos were generally invisible in the AA images, indicating that the microbubbles could not cross the placental barrier and therefore the risk of barrier permeability was low. The PA mode could not provide clear coronal images of the embryos because it was limited by the elevational resolution; however, PA mode may be used for other functional studies of mouse pregnancy, such as fetal oxygenation measurement and drug delivery monitoring [59-61].

From the US and AA images in Fig. 4, we can see half-moon-shaped organs close to the embryos. Since mature mouse placentas are established around mid-gestation ( E10.5) [62], these observed organs are most likely decidua before E10.5 and placentas afterwards. The basal decidua becomes progressively thinner over the pregnancy while placental villi within the labyrinth zones proliferate rapidly $[63,64]$. The AA signals of the placenta were weaker at E17, probably because the placentas were pushed to deeper regions with the developing embryos. The imaging depth of the AA mode can be improved by using microbubbles with smaller diameters, which can lower the pressure threshold needed to burst the bubbles in deep tissues $[65,66]$. The weaker placenta signals in PA images at E17 are likely due to the insufficient light penetration 
depth. Again, improving the light illumination geometry can help increase the imaging depth of the PA mode.

Acknowledgement: We thank Dr. Caroline Connor for editing the manuscript and Kun Xiang for technical support and useful discussion on animal experiments. This work was sponsored by National Institutes of Health (R01 EB028143, R01 NS111039, RF1 NS115581, R21 EB027304, R21EB027981, R43 CA243822, R43 CA239830, R44 HL138185); American Heart Association Collaborative Sciences Award (18CSA34080277); Chan Zuckerberg Initiative Grant on Deep Tissue Imaging 2020-226178 by Silicon Valley Community Foundation; All to J.Y.

Author contributions: J.Y., T.C. and R.G. conceptualized the study and oversaw the whole project. M.H. and G.O. constructed the hardware of US and AA modes. M.L. constructed the hardware of PA mode. N.B. and T.V. wrote software and synchronization sequences. C.M. contributed to the pregnant mouse experiment and photoswitching experiment. J.R. processed the AA and US images for small animal validation experiment. H.K. and J.L. developed the BIBDAH dye. L.K. and V.V. developed the photoswitching cell lines. Y.C., Q.H. and X.S. developed the pregnant mice. M.L. performed all the other experiments, processed the data and images. M.L. and J.Y. wrote the manuscript. N.B., M.H., G.O., J.R. T.C. and R.G. edited the manuscript.

\section{Online Methods:}

PAUSAT scanning configuration: In the US and AA mode, a field of view of $\sim 40 \mathrm{~mm}$ by 20 $\mathrm{mm}$ (lateral by elevational) was scanned by the wobbler with a scanning step size of $0.2 \mathrm{~mm}$ along the elevational dimension and the pixel size is $0.05 \mathrm{~mm}$ along the lateral dimension. The width of the wobbler field of view is $\sim 17 \mathrm{~mm}$ and therefore 3 passes were taken and stitched to span $40 \mathrm{~mm}$. In the AA mode, the lateral scanning was repeated 5 times and averaged to enhance the image quality. It took $\sim 5$ min to acquire a 3D volumetric US data and 30 min for a 3D AA data. For the US and AA mode, 3D images were reconstructed using a pixel-based volume reconstruction method and compounding for overlapped pixels [67]. In PA mode, the linear array was scanned elevationally with a step size of $0.2 \mathrm{~mm}$ and a scanning length of $40 \mathrm{~mm}$. Five repeated frames are taken at each location for averaging. It took $\sim 5$ mins to acquire a 3D PA data. The optical pulse energy from the fiber output was $~ 20 \mathrm{~mJ}$ at $1064 \mathrm{~nm}$, with a spot size of 10 
$\mathrm{mm}$ by $40 \mathrm{~mm}$ on the sample surface. The axial cross-sectional PA images were reconstructed in real time and 3D rendering images were processed offline using Matlab. For the PA mode, the back-projection reconstruction method was performed for each cross-sectional image [68, 69].

Phantom preparation: In the AA result in Fig. 2(a), microbubbles (Sonovol, Inc.; Diameter: 5 $\mu \mathrm{m}$ ) were infused into the plastic tube (inner diameter, $200 \mu \mathrm{m}$; outer diameter $300 \mu \mathrm{m}$ ) at the rate of $1 \times 10^{8}$ bubbles/min continuously with a catheter, which was driven by an automatic syringe pump to mimic the blood circulation. In the PA results of Fig. 2(d), the lab-made BIBDAH dye was prepared with four different concentrations $(0.5 \mathrm{mg} / \mathrm{mL}, 0.2 \mathrm{mg} / \mathrm{mL}, 0.1$ $\mathrm{mg} / \mathrm{mL}, 0.05 \mathrm{mg} / \mathrm{mL}$ ) diluted with PBS [36]. The dye solutions were injected into transparent plastic tubes (inner diameter: $200 \mu \mathrm{m}$; outer diameter: $300 \mu \mathrm{m}$ ). A fifth tube was filled with whole bovine blood (Quad Five, Inc.). The tubes were sandwiched by two pieces of thin agar sheets and placed on top of the imaging window of the PAUSAT system. The imaging window was then covered by a thin layer of distilled water as the ultrasound coupling medium.

Animal preparation: The protocol of all the animal experiments was proved by the institutional animal care and use committee of Duke University. Female BALB/c mice (8 weeks old; 20-30 grams in weight) were used for all experiments. The hair on the mouse chest, abdomen or back was shaved and depilated as needed. The mice were then anesthetized with $1.5 \% \mathrm{v} / \mathrm{v}$ isoflurane and placed on the imaging window of the PAUSAT system with a 1-mm-thick agar pad underneath. This agar pad served as a spacer to separate the PA and US signals from the mouse skin surface and the imaging window membrane. Water was used to couple the membrane, the agar pad and the mouse skin. A heating lamp was used to maintain mouse body temperature at $37^{\circ} \mathrm{C}$. For all the AA imaging, a total of $200 \mu \mathrm{L}$ microbubbles with the concentration of $2 \times 10^{9}$ $/ \mathrm{ml}$ were injected via the tail vein with a 27 -gauge syringe. For the PA liver imaging shown in Fig. 3, Matrigel mixed with the BIBDAH dye was implanted around the liver region subcutaneously to create an artificial tumor with a volume of $15 \mathrm{~mm}^{3}$. For PA dye metabolism imaging shown in Fig. 5, $200 \mu \mathrm{L}$ BIBDAH dye with the concentration of $0.5 \mathrm{mg} / \mathrm{mL}$ was injected via the tail vein, and the PA imaging were performed every hour.

Pregnant mouse preparation: CD-1 IGS mice of $\sim 8$ weeks old were used for timed breeding by checking vaginal plugs. To prepare the pregnant mice, female mice at the proestrus phase were placed together with male mice for mating. The females were then checked in the following 
morning at 8-9 am for vaginal plug. When a plug was seen, they were separated and the day when the plug was found was considered as the first day of gestation. The embryos were also staged in days of gestation, and the morning when the vaginal plug was detected was defined as E0.5. The pregnant mice were imaged from E8.5.

Photoswitching imaging: Bphpl-expressing 4T1 cells were grown in DMEM supplemented with $10 \%$ FBS and $1 \%$ penicillin-streptomycin mixture at $37{ }^{\circ} \mathrm{C}$ in $5 \% \mathrm{CO}_{2}$ atmosphere. The $\mathrm{BALB} / \mathrm{c}$ mouse was placed under anesthesia with $1.5 \%$ isoflurane and BphPl-expressing 4T1 cells $\left(4 \times 10^{4}\right)$ were injected subcutaneously under the $4^{\text {th }}$ mammary fat pad. The mouse was imaged when the tumor diameter was $\sim 5 \mathrm{~mm}$, as measured by calipers. For all photoswitching studies, the switching settings were the same. A total of $8 \mathrm{~s}$ of $635 \mathrm{~nm} \mathrm{CW}$ light was used for switching on the Bphp1 protein, followed by a total of $8 \mathrm{~s}$ of $750 \mathrm{~nm}$ pulse light for both PA imaging and switching off the protein. We acquired 24 switching cycles and averaged the images accordingly. The differential images were obtained by subtracting the OFF-state images from the ON-state images $[29,70]$.

\section{References}

1. Cunha, L., et al., Preclinical imaging: an essential ally in modern biosciences. Mol Diagn Ther, 2014. 18(2): p. 153-73.

2. Lewis, J.S., et al., Small animal imaging. current technology and perspectives for oncological imaging. Eur J Cancer, 2002. 38(16): p. 2173-88.

3. Pandit, P., et al., The utility of micro-CT and MRI in the assessment of longitudinal growth of liver metastases in a preclinical model of colon carcinoma. Acad Radiol, 2013. 20(4): p. 430-9.

4. Vaquero, J.J. and P. Kinahan, Positron Emission Tomography: Current Challenges and Opportunities for Technological Advances in Clinical and Preclinical Imaging Systems. Annu Rev Biomed Eng, 2015. 17: p. 385-414.

5. Hutchinson, J.C., et al., Early clinical applications for imaging at microscopic detail: microfocus computed tomography (micro-CT). British Journal of Radiology, 2017. 90(1075).

6. Jiang, Y., et al., Micro CT and Micro MR imaging of 3D architecture of animal skeleton. J Musculoskelet Neuronal Interact, 2000. 1(1): p. 45-51.

7. Ashton, J.R., J.L. West, and C.T. Badea, In vivo small animal micro-CT using nanoparticle contrast agents. Frontiers in Pharmacology, 2015. 6.

8. Judenhofer, M.S. and S.R. Cherry, Applications for preclinical PET/MRI. Semin Nucl Med, 2013. 43(1): p. 19-29.

9. Lee, C.L., et al., Assessing cardiac injury in mice with dual energy-microCT, 4D-microCT, and microSPECT imaging after partial heart irradiation. Int J Radiat Oncol Biol Phys, 2014. 88(3): p. 686-93.

10. Clark, D.P. and C.T. Badea, Micro-CT of rodents: state-of-the-art and future perspectives. Phys Med, 2014. 30(6): p. 619-34. 
11. Cavanaugh, D., et al., In vivo respiratory-gated micro-CT imaging in small-animal oncology models. Mol Imaging, 2004. 3(1): p. 55-62.

12. Ambrosini, V., et al., Small animal PET in oncology: the road from bench to bedside. Cancer Biother Radiopharm, 2009. 24(2): p. 277-85.

13. Hoyer, C., et al., Advantages and challenges of small animal magnetic resonance imaging as a translational tool. Neuropsychobiology, 2014. 69(4): p. 187-201.

14. Darne, C., Y. Lu, and E.M. Sevick-Muraca, Small animal fluorescence and bioluminescence tomography: a review of approaches, algorithms and technology update. Phys Med Biol, 2014. 59(1): p. R1-64.

15. Davis, S.C. and K.M. Tichauer, Small-Animal Imaging Using Diffuse Fluorescence Tomography. Methods Mol Biol, 2016. 1444: p. 123-37.

16. Henriquez, N.V., et al., Advances in optical imaging and novel model systems for cancer metastasis research. Clin Exp Metastasis, 2007. 24(8): p. 699-705.

17. Puaux, A.L., et al., A comparison of imaging techniques to monitor tumor growth and cancer progression in living animals. Int J Mol Imaging, 2011. 2011: p. 321538.

18. Greco, A., et al., Ultrasound biomicroscopy in small animal research: applications in molecular and preclinical imaging. J Biomed Biotechnol, 2012. 2012: p. 519238.

19. Hyvelin, J.M., et al., Use of ultrasound contrast agent microbubbles in preclinical research: recommendations for small animal imaging. Invest Radiol, 2013. 48(8): p. 570-83.

20. Sun, L., et al., A high-frame rate duplex ultrasound biomicroscopy for small animal imaging in vivo. IEEE Trans Biomed Eng, 2008. 55(8): p. 2039-49.

21. Dayton, P.A., et al., The implementation of acoustic angiography for microvascular and angiogenesis imaging. Annu Int Conf IEEE Eng Med Biol Soc, 2014. 2014: p. 4283-5.

22. Newsome, I.G. and P.A. Dayton, Visualization of Microvascular Angiogenesis Using DualFrequency Contrast-Enhanced Acoustic Angiography: A Review. Ultrasound Med Biol, 2020. 46(10): p. 2625-2635.

23. Shelton, S.E., et al., Molecular Acoustic Angiography: A New Technique for High-resolution Superharmonic Ultrasound Molecular Imaging. Ultrasound Med Biol, 2016. 42(3): p. 769-81.

24. Wang, L.V. and S. Hu, Photoacoustic tomography: in vivo imaging from organelles to organs. Science, 2012. 335(6075): p. 1458-62.

25. Wang, L.V. and J. Yao, A practical guide to photoacoustic tomography in the life sciences. Nat Methods, 2016. 13(8): p. 627-38.

26. Beard, P., Biomedical photoacoustic imaging. Interface Focus, 2011. 1(4): p. 602-31.

27. Li, M., Y. Tang, and J. Yao, Photoacoustic tomography of blood oxygenation: A mini review. Photoacoustics, 2018. 10: p. 65-73.

28. Li, M.C., et al., Sound Out the Deep Colors: Photoacoustic Molecular Imaging at New Depths. Molecular Imaging, 2020. 19.

29. Yao, J.J., et al., Multiscale photoacoustic tomography using reversibly switchable bacterial phytochrome as a near-infrared photochromic probe. Nature Methods, 2016. 13(1): p. 67-+.

30. Gessner, R.C., et al., Acoustic angiography: a new imaging modality for assessing microvasculature architecture. Int J Biomed Imaging, 2013. 2013: p. 936593.

31. Gessner, R.C., S.R. Aylward, and P.A. Dayton, Mapping Microvasculature with Acoustic Angiography Yields Quantifiable Differences between Healthy and Tumor-bearing Tissue Volumes in a Rodent Model. Radiology, 2012. 264(3): p. 733-740.

32. Czernuszewicz, T.J., et al., A new preclinical ultrasound platform for widefield 3D imaging of rodents. Rev Sci Instrum, 2018. 89(7): p. 075107.

33. Nishiyama, M., et al., Ring-array photoacoustic tomography for imaging human finger vasculature. J Biomed Opt, 2019. 24(9): p. 1-12. 
34. Laufer, J., et al., Three-dimensional noninvasive imaging of the vasculature in the mouse brain using a high resolution photoacoustic scanner. Appl Opt, 2009. 48(10): p. D299-306.

35. Taruttis, A., et al., Optoacoustic Imaging of Human Vasculature: Feasibility by Using a Handheld Probe. Radiology, 2016. 281(1): p. 256-63.

36. Kilian, H.I., et al., Facile formulation of a long-wavelength cyanine for optical imaging in the second near-infrared window. Biomater Sci, 2020. 8(15): p. 4199-4205.

37. Ku, G. and L.V. Wang, Deeply penetrating photoacoustic tomography in biological tissues enhanced with an optical contrast agent. Opt Lett, 2005. 30(5): p. 507-9.

38. Zhang, Q., et al., Gold nanoparticles as a contrast agent for in vivo tumor imaging with photoacoustic tomography. Nanotechnology, 2009. 20(39): p. 395102.

39. Bosschaart, N., et al., A literature review and novel theoretical approach on the optical properties of whole blood. Lasers Med Sci, 2014. 29(2): p. 453-79.

40. Luke, G.P., D. Yeager, and S.Y. Emelianov, Biomedical applications of photoacoustic imaging with exogenous contrast agents. Ann Biomed Eng, 2012. 40(2): p. 422-37.

41. Demitri, C., et al., Hydrogel based tissue mimicking phantom for in-vitro ultrasound contrast agents studies. J Biomed Mater Res B Appl Biomater, 2008. 87(2): p. 338-45.

42. Huang, Q., et al., Intravital imaging of mouse embryos. Science, 2020. 368(6487): p. 181-186.

43. Mu, J.W., et al., In vivo quantification of embryonic and placental growth during gestation in mice using micro-ultrasound. Reproductive Biology and Endocrinology, 2008. 6.

44. Roberts, V.H.J. and A.E. Frias, Contrast-enhanced ultrasound for the assessment of placental development and function. Biotechniques, 2020. 69(5): p. 392-399.

45. Preisser, S., et al., Vessel orientation-dependent sensitivity of optoacoustic imaging using a linear array transducer. J Biomed Opt, 2013. 18(2): p. 26011.

46. Mishra, K., et al., Multiplexed whole-animal imaging with reversibly switchable optoacoustic proteins. Sci Adv, 2020. 6(24): p. eaaz6293.

47. Wang, Y.H., et al., Review of methods to improve the performance of linear array-based photoacoustic tomography. Journal of Innovative Optical Health Sciences, 2020. 13(2).

48. Xia, J., et al., Three-dimensional photoacoustic tomography based on the focal-line concept. J Biomed Opt, 2011. 16(9): p. 090505.

49. Fehm, T.F., et al., In vivo whole-body optoacoustic scanner with real-time volumetric imaging capacity. Optica, 2016. 3(11): p. 1153-1159.

50. Wu, Y.L., et al., Emerging contrast agents for multispectral optoacoustic imaging and their biomedical applications. Chemical Society Reviews, 2021. 50(14): p. 7924-7940.

51. Mattes, D., et al., PET-CT image registration in the chest using free-form deformations. leee Transactions on Medical Imaging, 2003. 22(1): p. 120-128.

52. Streeter, J.E., et al., Improving sensitivity in ultrasound molecular imaging by tailoring contrast agent size distribution: in vivo studies. Mol Imaging, 2010. 9(2): p. 87-95.

53. Shashank Sirsi, J.F., Mark Borden, Shunichi Homma, High-frequency ultrasound imaging of sizeisolated microbubbles in mice. IEEE International Ultrasonics Symposium Proceedings, 2009. 2023(Sept. 2009).

54. Feshitan, J.A., et al., Microbubble size isolation by differential centrifugation. J Colloid Interface Sci, 2009. 329(2): p. 316-24.

55. Kimmel, E., Cavitation bioeffects. Crit Rev Biomed Eng, 2006. 34(2): p. 105-61.

56. Yuan, F., C. Yang, and P. Zhong, Cell membrane deformation and bioeffects produced by tandem bubble-induced jetting flow. Proc Natl Acad Sci U S A, 2015. 112(51): p. E7039-47.

57. Annemieke van Wamel, A.B., Folkert ten Cate, Nico de Jong, Effects of Diagnostic Ultrasound Parameters on Molecular Uptake and Cell Viability. Proceedings of the International Ultrasonics Symposium, 2002. 8-11(October 2002). 
58. Xie, F., et al., Effects of transcranial ultrasound and intravenous microbubbles on blood brain barrier permeability in a large animal model. Ultrasound Med Biol, 2008. 34(12): p. 2028-34.

59. Bayer, C.L., et al., Ultrasound-guided spectral photoacoustic imaging of hemoglobin oxygenation during development. Biomed Opt Express, 2017. 8(2): p. 757-763.

60. Basak, K., et al., Non-invasive determination of murine placental and foetal functional parameters with multispectral optoacoustic tomography. Light Sci Appl, 2019. 8: p. 71.

61. Huda, K., et al., Spherical-view photoacoustic tomography for monitoring in vivo placental function. Photoacoustics, 2020. 20: p. 100209.

62. Woods, L., V. Perez-Garcia, and M. Hemberger, Regulation of Placental Development and Its Impact on Fetal Growth-New Insights From Mouse Models. Frontiers in Endocrinology, 2018. 9.

63. Turner, R.J. and B.A. Carroll, Ultrasound appearance of decidual reaction in the uterus during normal pregnancy. AJR Am J Roentgenol, 1979. 133(3): p. 493-6.

64. Cross, J.C., et al., Branching morphogenesis during development of placental villi. Differentiation, 2006. 74(7): p. 393-401.

65. Chomas, J.E., et al., Threshold of fragmentation for ultrasonic contrast agents. J Biomed Opt, 2001. 6(2): p. 141-50.

66. Newsome, I.G., T.M. Kierski, and P.A. Dayton, Assessment of the Superharmonic Response of Microbubble Contrast Agents for Acoustic Angiography as a Function of Microbubble Parameters. Ultrasound Med Biol, 2019. 45(9): p. 2515-2524.

67. Lasso, A., et al., PLUS: open-source toolkit for ultrasound-guided intervention systems. IEEE Trans Biomed Eng, 2014. 61(10): p. 2527-37.

68. $\mathrm{Xu}, \mathrm{M}$. and L.V. Wang, Universal back-projection algorithm for photoacoustic computed tomography. Phys Rev E Stat Nonlin Soft Matter Phys, 2005. 71(1 Pt 2): p. 016706.

69. Wang, Z., J. Li, and R. Wu, Time-delay- and time-reversal-based robust Capon beamformers for ultrasound imaging. IEEE Trans Med Imaging, 2005. 24(10): p. 1308-22.

70. Li, L., et al., Small near-infrared photochromic protein for photoacoustic multi-contrast imaging and detection of protein interactions in vivo. Nat Commun, 2018. 9(1): p. 2734. 
(a)

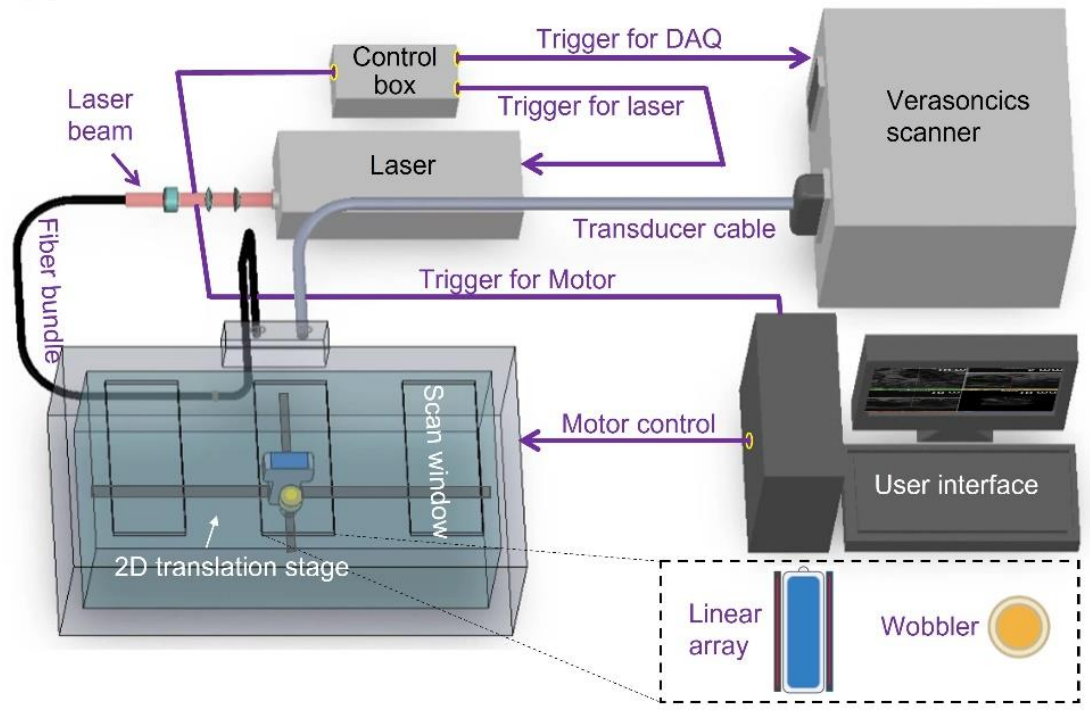

(c)
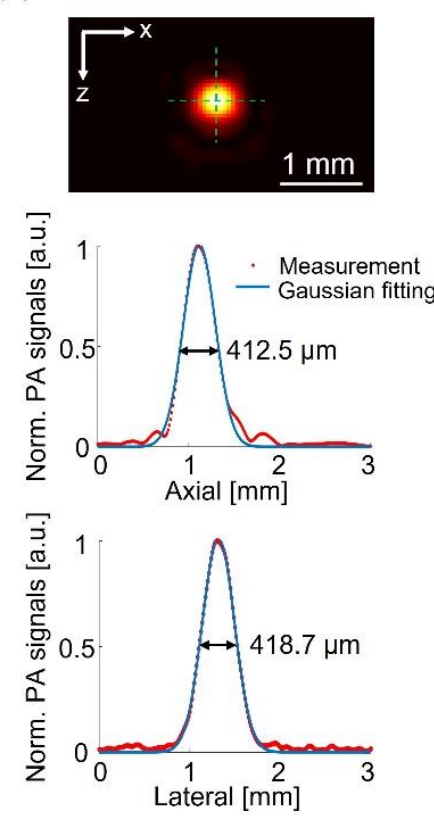

(d)
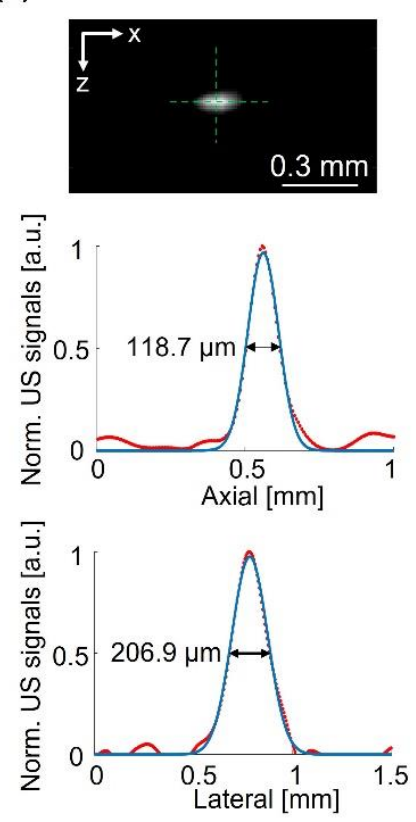

(b)
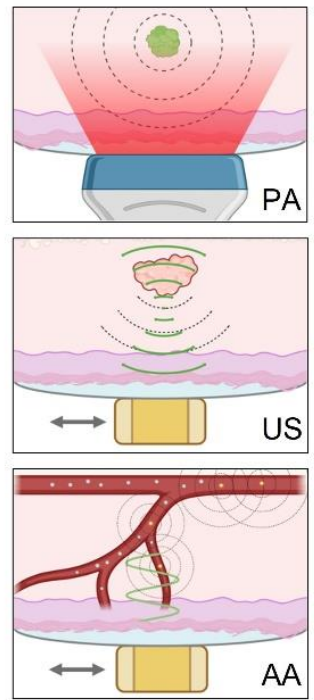

(e)
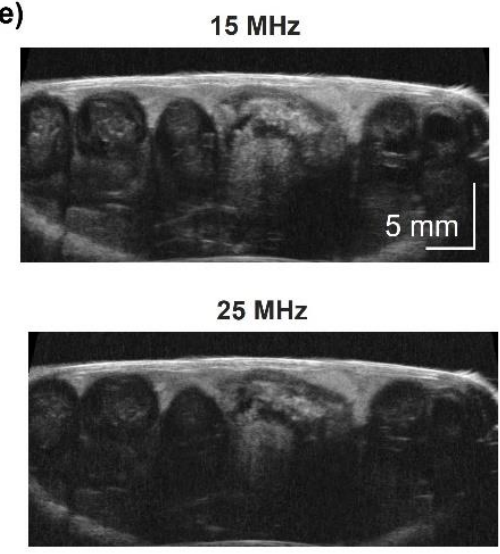

$35 \mathrm{MHz}$

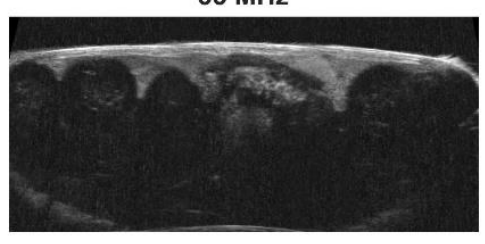

(a) Schematic of the PAUSAT system, showing the key acoustic, optical and mechanical components. (b) Imaging principles of the PA, US, and AA modes. In the PA mode, the pulsed laser light is delivered by the optical fiber bundle and the resultant acoustic waves are detected by the linear transducer array; In the US mode, the high-frequency inner-element of the focused

564 wobbler transducer transmits the acoustic waves and detects the reflected echo signals; In the AA mode, the low-frequency outer-element of the wobbler transmits a strong acoustic pulse to burst the flowing microbubbles in the blood vessels, and the high-frequency inner-elements detects the 
567 bursting signals from the bubbles. (c) Spatial resolution measurement of the PA mode. (d)

568 Spatial resolution measurement of the US mode. (e) Comparison of the penetration depth of the 569 US mode at three different frequencies.

(a)

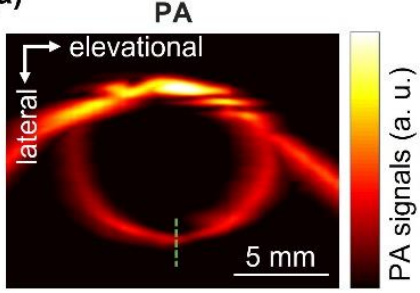

(b)
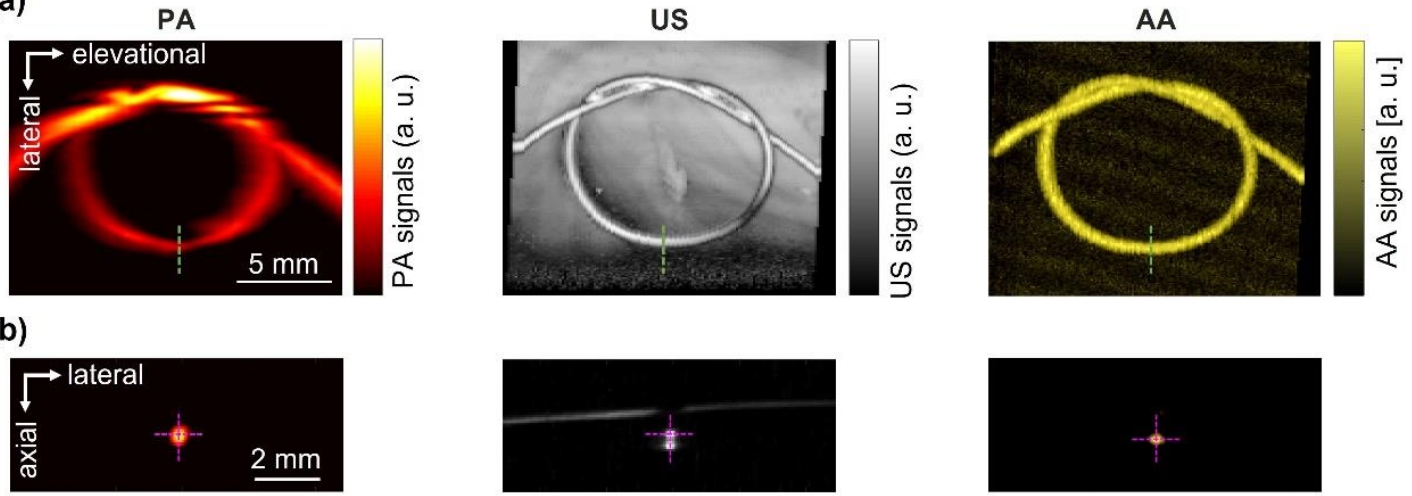

(c)
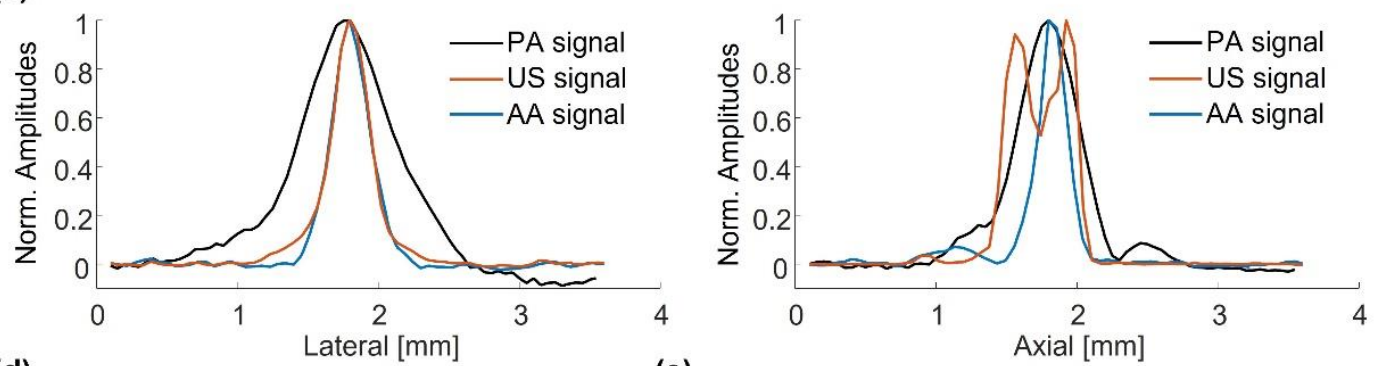

(d)

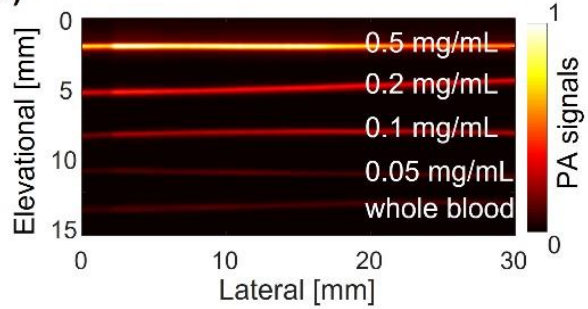

(e)

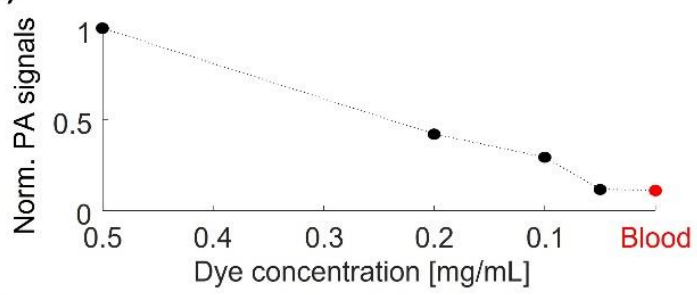

(f)
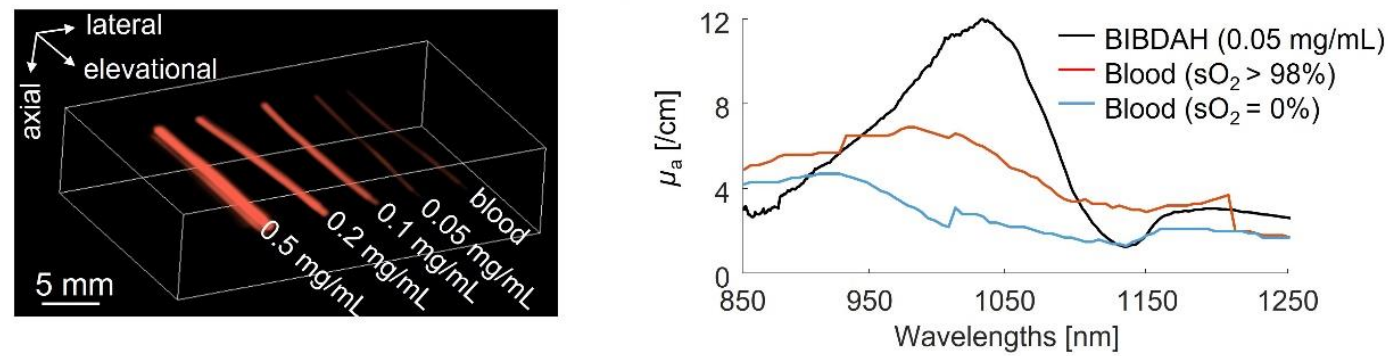

570

571 Fig. 2. PAUSAT system validation on phantoms. (a) Images of a tube knot filled with

572 BIBDAH, water, and microbubbles with PA, US, and AA, respectively. (b) Cross-sectional 573 images of the tube along the green-dotted lines in (a). (c) Lateral and axial signal profiles along 574 the magenta dotted lines in (b). (d) Top-view projection PA image and 3D rendering of tubes 
575 filled with blood and BIBDAH dye at different concentrations. (e) Quantitative PA signals from

576 blood and dyes. (f) Optical absorption spectra of BIBDAH at $50 \mu \mathrm{g} / \mathrm{ml}$, oxygenated and 577 deoxygenated blood.

(a)

(b)
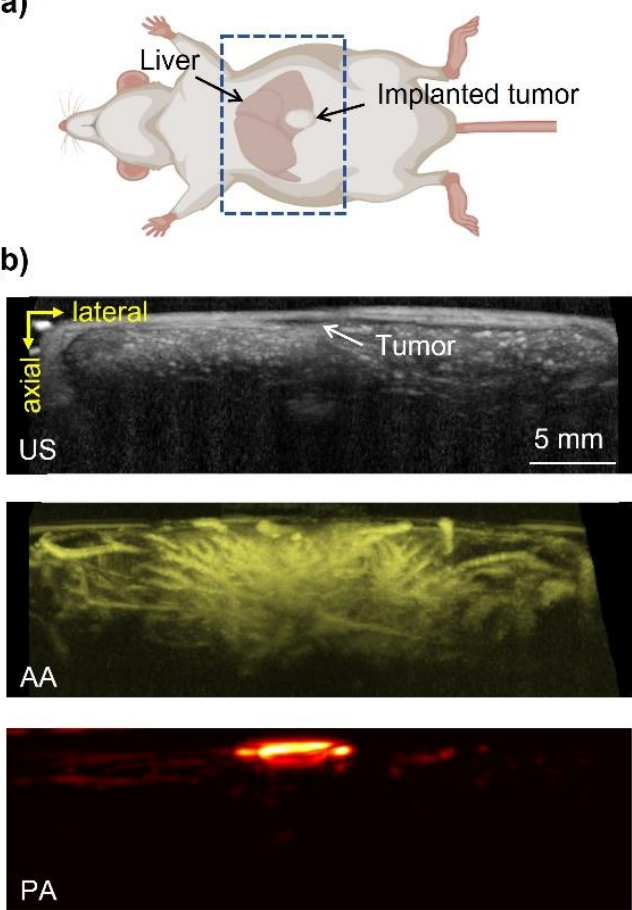

(d)

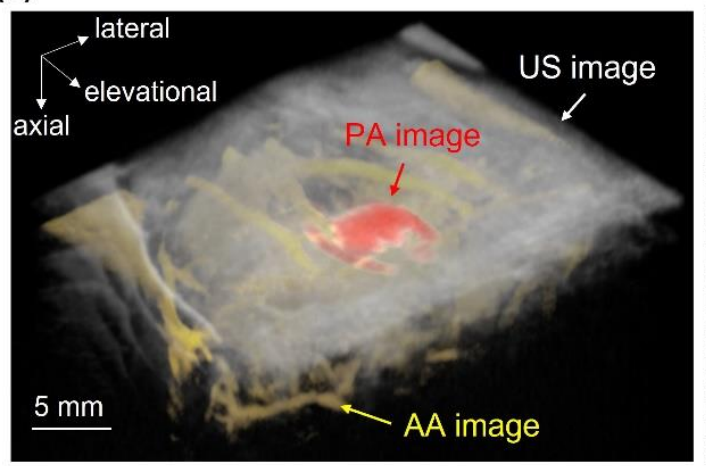

(e)
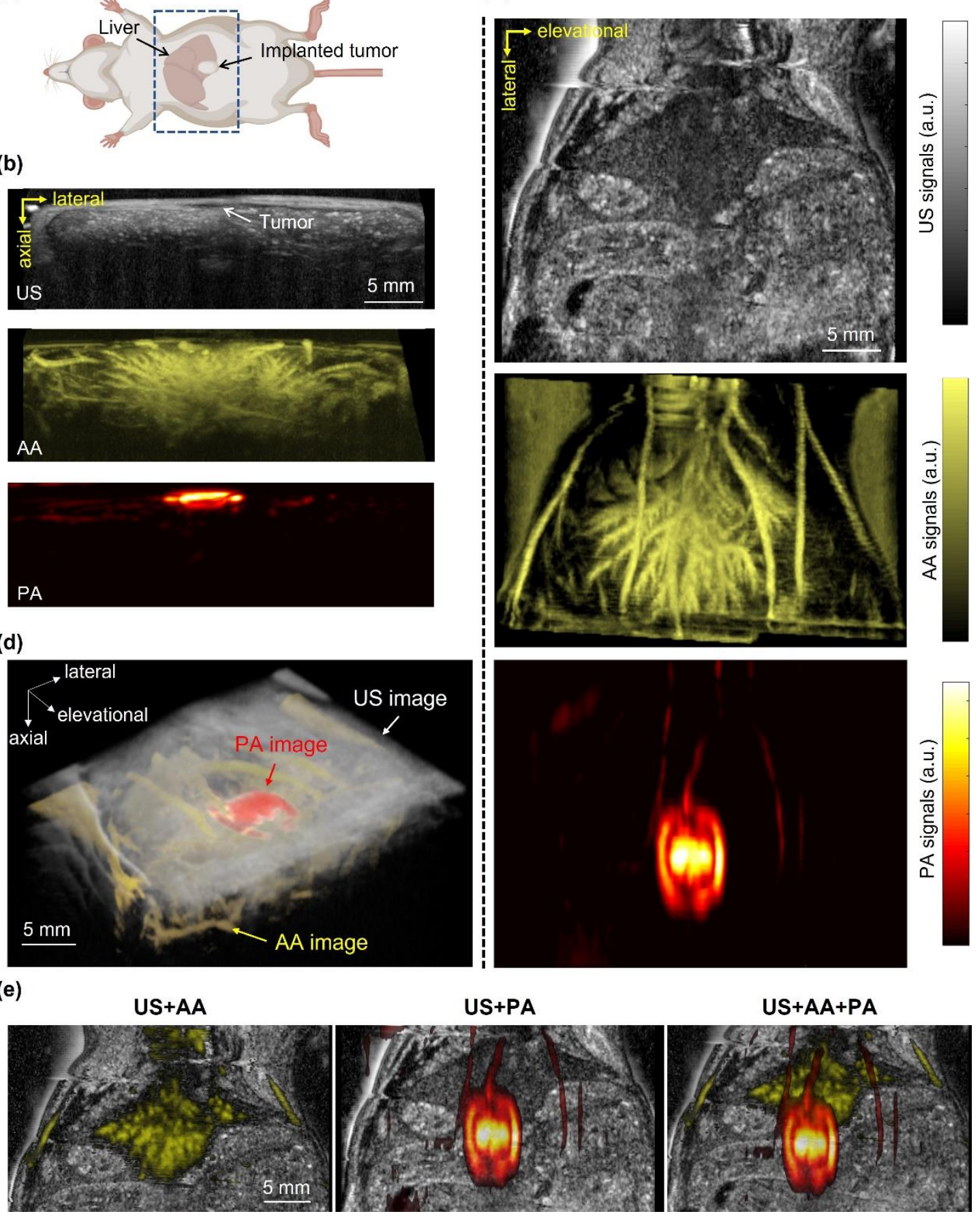

579 Fig. 3. PAUSAT system validation on small animals. (a) The implantation position of the 
581 by the PA, US, and AA mode. (c) Coronal images of the liver region by the PA, US, and AA 582 mode. US shows one slice where the liver was located while AA and PA show the maximum 583 amplitude projections. (d) Three-dimensional rendering of the superimposed PA (in hot color), 584 US (in gray color), and AA (in yellow color) images. (e) Superimposed images of different 585 imaging modes showing the relative positions of the targets.

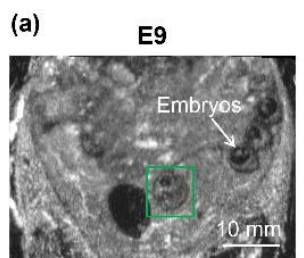

(b)

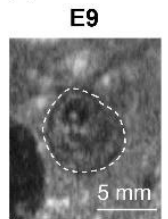

(d)

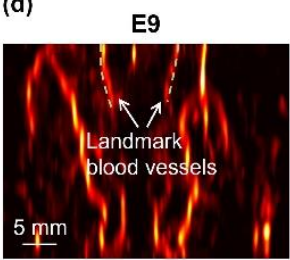

(e)

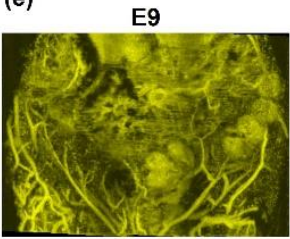

E11
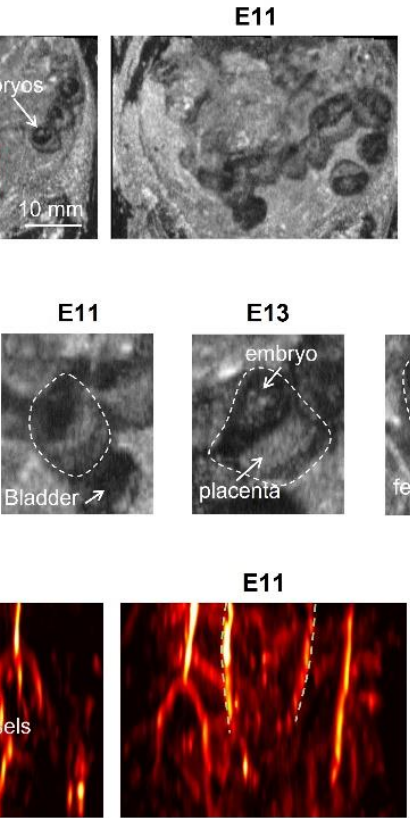

E13

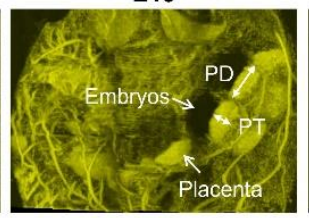

E13

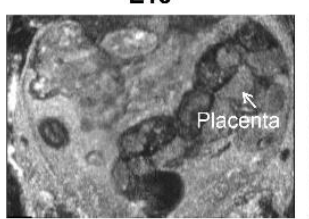

E15

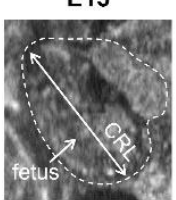

E15

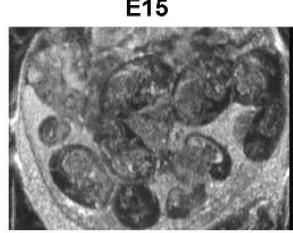

(c)

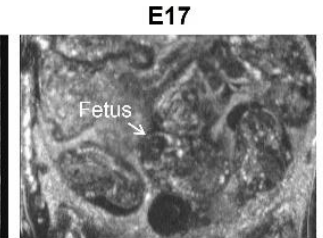

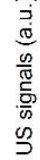
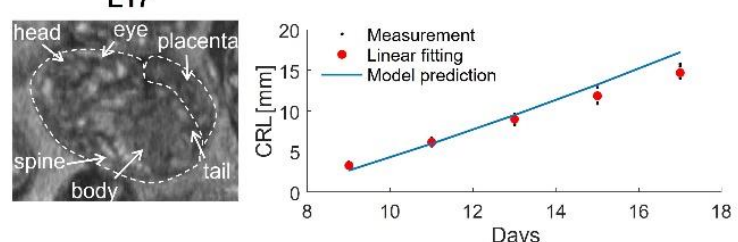

E17
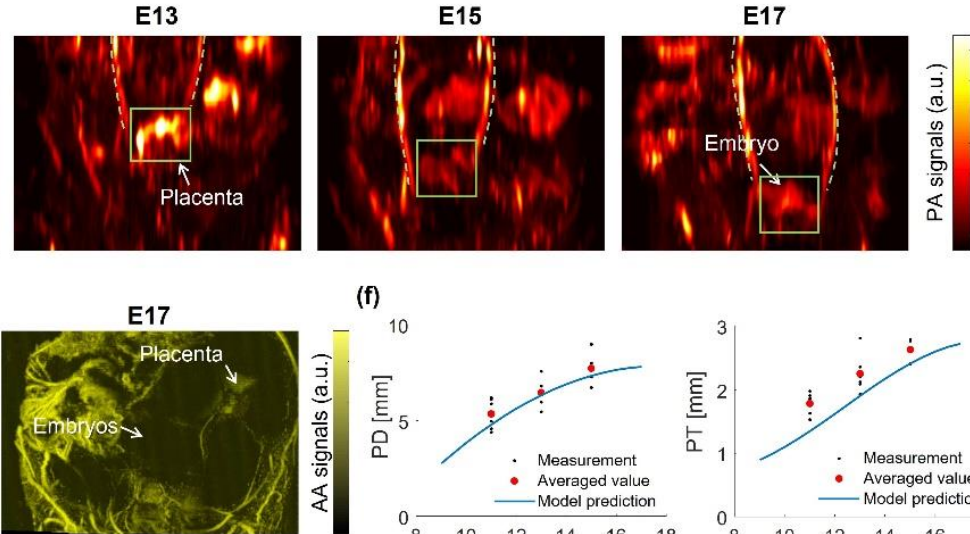

${ }_{-}^{(f)} 10$

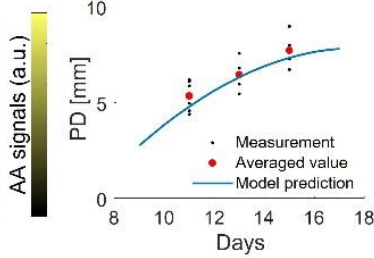

Fig 4. Longitudinal imaging of mouse pregnancy by PAUSAT. (a) Longitudinal US images of a pregnant mouse at E9, E11, E13, E15 and E17. (b) Close-up images of a single embryo from E9 to E17. (c) Embryo's crown-rump length measured from the US images (black dots), the

590 linear fitting (red dots), and the model prediction (blue curve). (d) Longitudinal PA images of the 591 pregnant mouse. (e) Longitudinal AA images of the maternal vasculature and placentas. (f) 592 Placental diameter (PD) and placental thickness (PT) measurements from the US and AA images 593 for individual embryos (black dots), the averaged values (red dots), and the model prediction 594 (blue curves). 
(a)

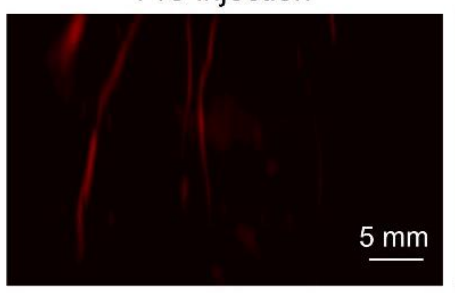

$2 \mathrm{~h}$

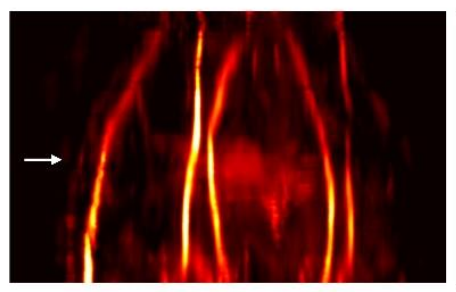

(b)

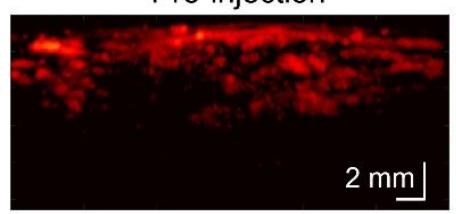

(c)

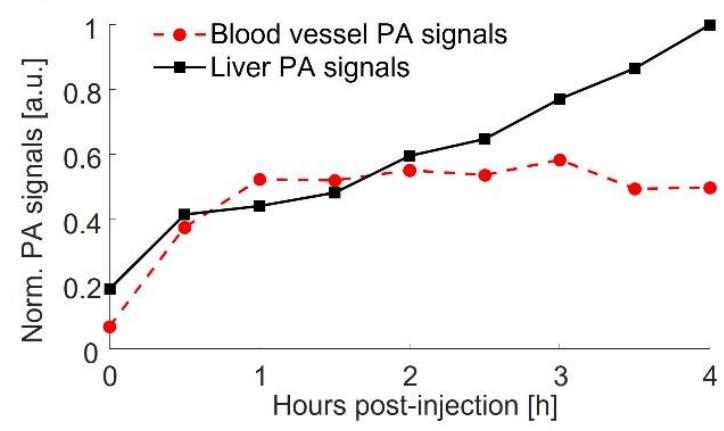

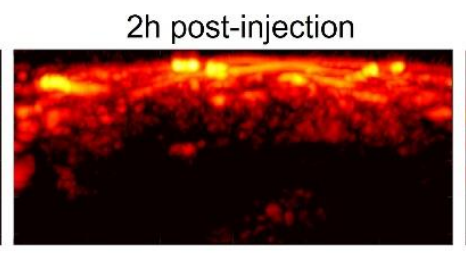

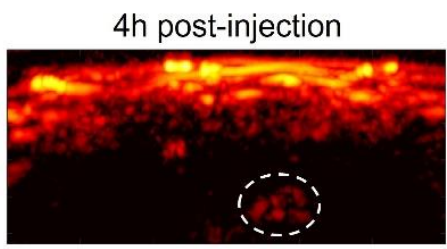

$0.5 \mathrm{~h}$

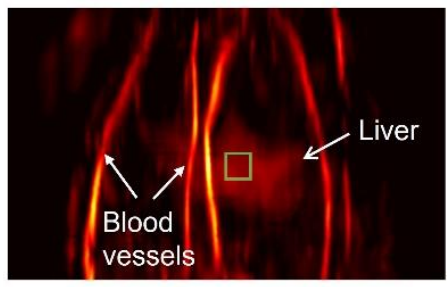

$3 \mathrm{~h}$
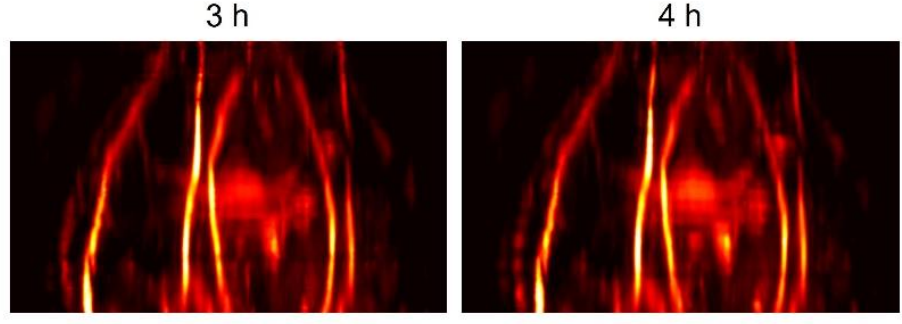

(d)
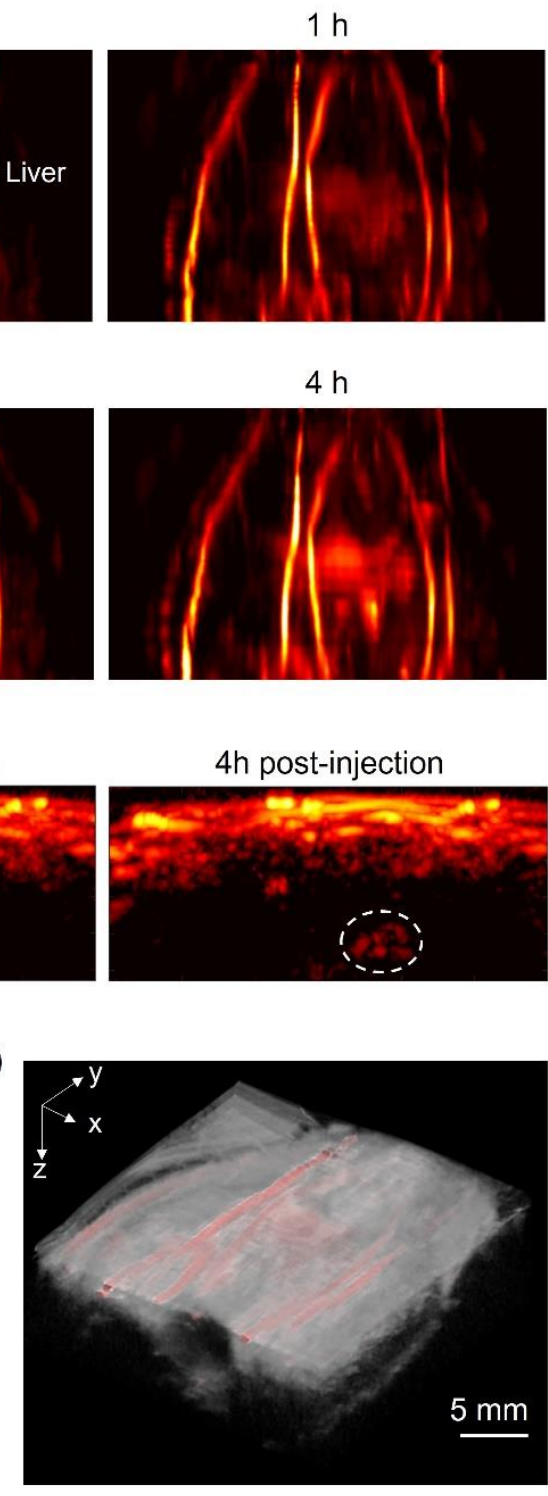

596 Fig. 5. Tracking the biodistribution of an NIR-II dye. (a) PA images of the mouse liver region

597 before and after the injection of an NIR-II dye BIBDAH. (b) Axial cross-sectional images of the

598 liver region indicated by the white arrow in (a). (c) PA signal dynamics in the major blood 599 vessels and in the liver region as indicated by the green square in (a). (d) 3D rendering of the 600 superimposed US (shown in gray) and PA (shown in color) image of the mouse 4 hours post601 injection. 
(a)

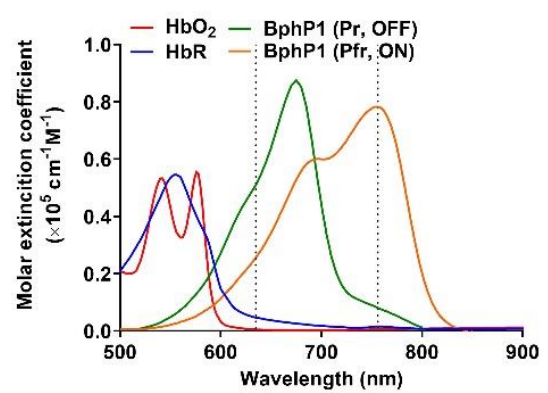

(d)

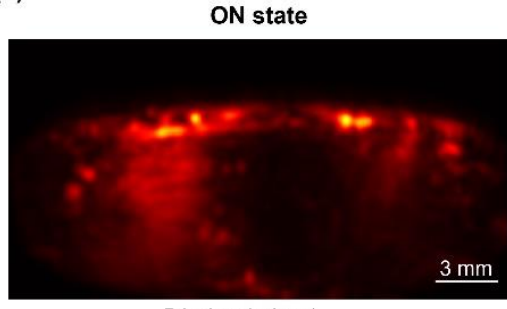

PA signals (a.u.)

(f)

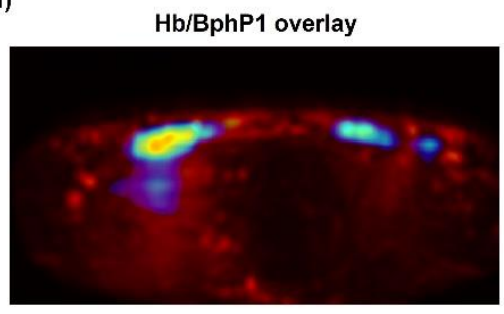

(b)

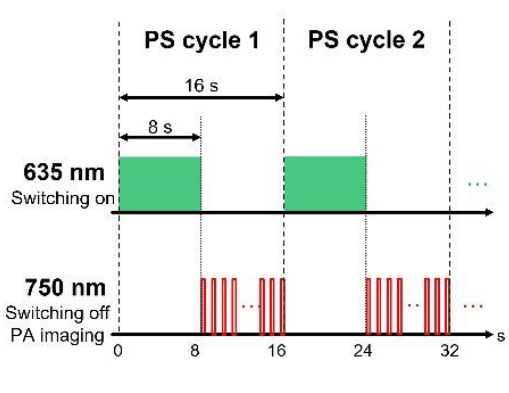

OFF state

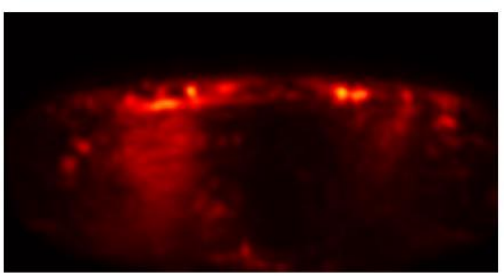

Ultrasound/BphP1 overlay

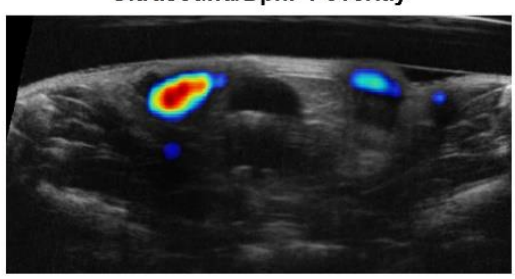

(c)
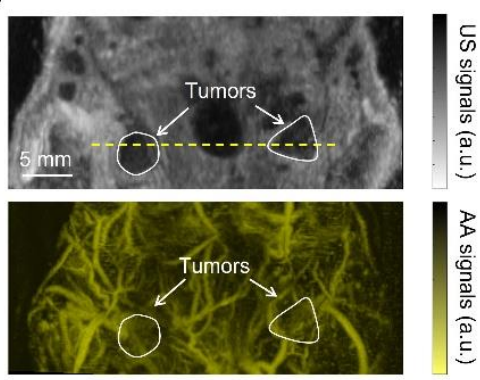

(e)

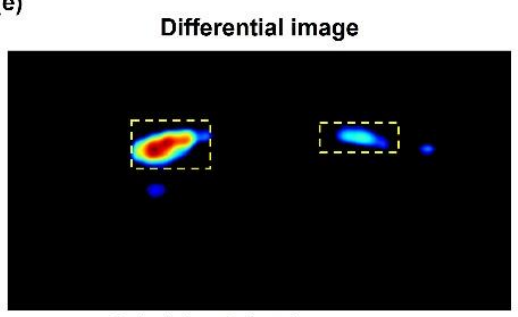

Bphp1 signals (a.u.)

(g)

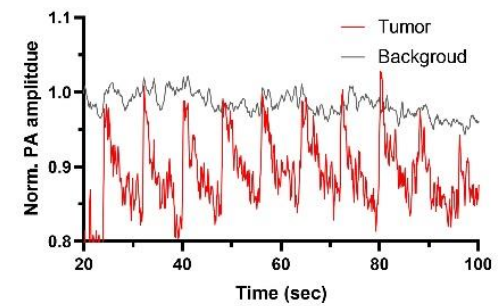

Fig. 6. Detecting reversibly photoswitching tumor in vivo. (a) Molecular extinction coefficients of oxygenated hemoglobin $\left(\mathrm{HbO}_{2}\right.$, red curve), deoxygenated hemoglobin ( $\mathrm{Hb}$, blue curve), ON state Bphpl (orange curve) and OFF state Bphpl (green curve) from $500 \mathrm{~nm}$ to 900 nm. (b) PA laser excitation sequence used for photoacoustic imaging and photoswitching Bphpl.

607 The PA images were acquired at $750 \mathrm{~nm}$. (c) Coronal-view US and AA images of the mouse 608 tumor region. The circles indicate the positions of implanted tumors. (d) Axial cross-sectional 609 PA images at the ON and OFF state of the Bphpl tumor. The position of the cross-section is 610 indicated in the US image in (c). (e) Differential PA image between the ON and OFF state, 611 showing the tumor only. (f) The differential image overlayed with PA and US images. (g) PA 612 signal changes from tumor and blood over multiple photoswitching cycles. 
(a)

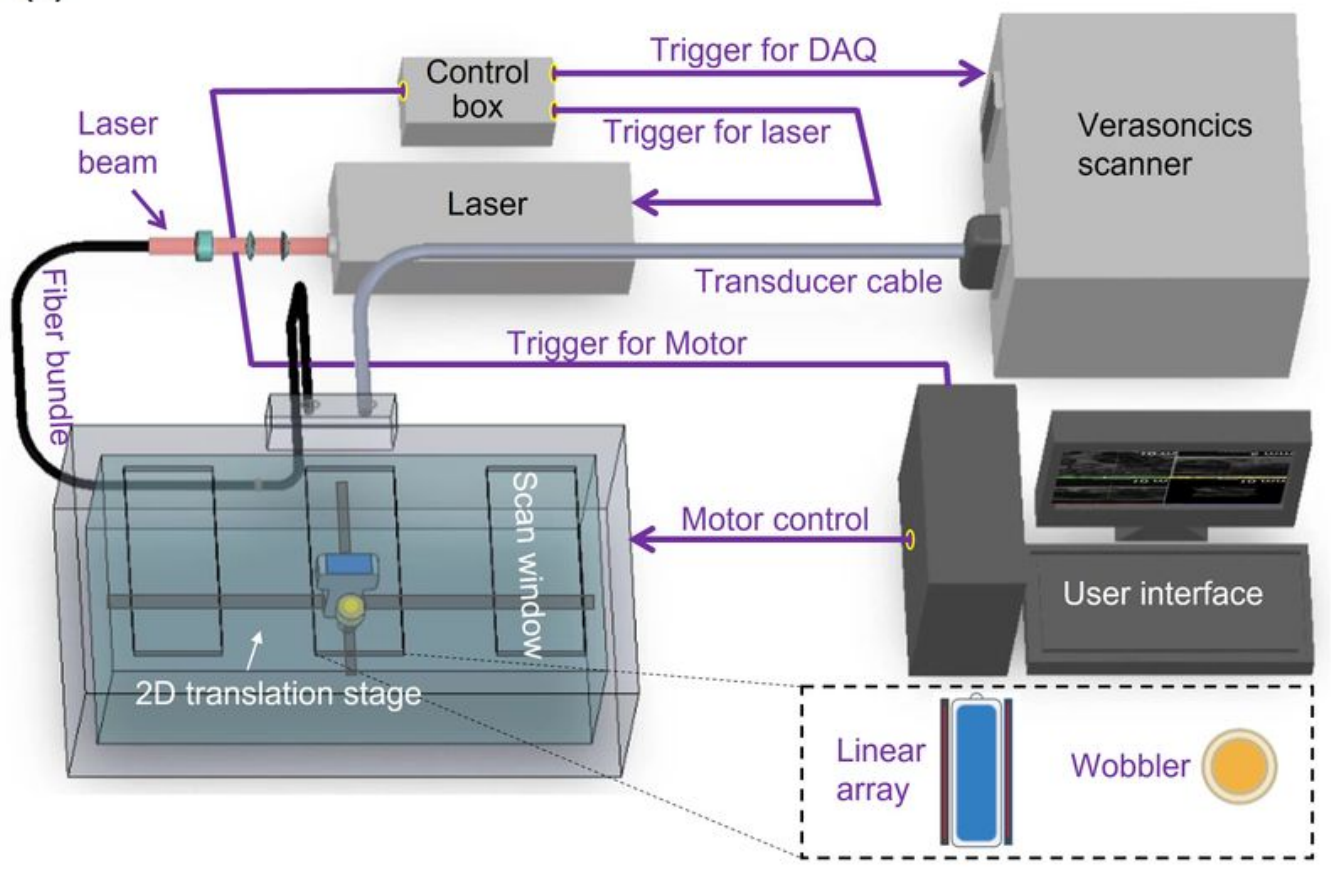

(b)
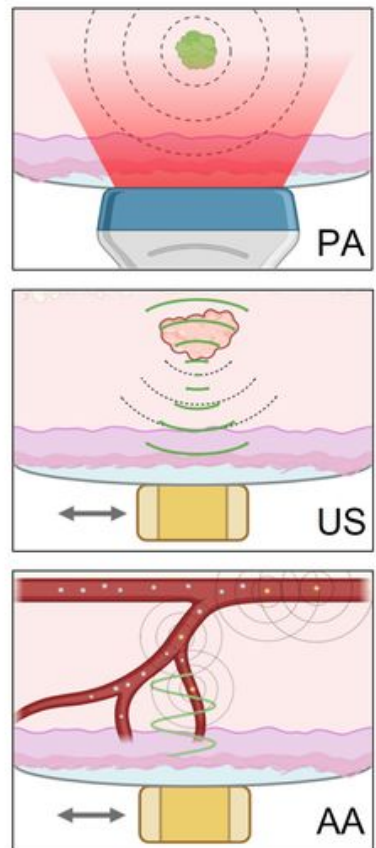

(c)
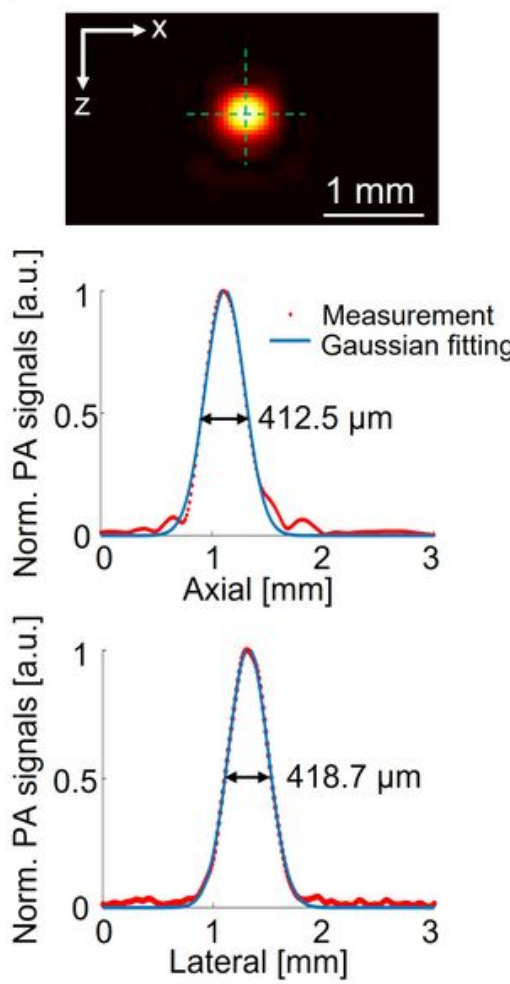

(d)
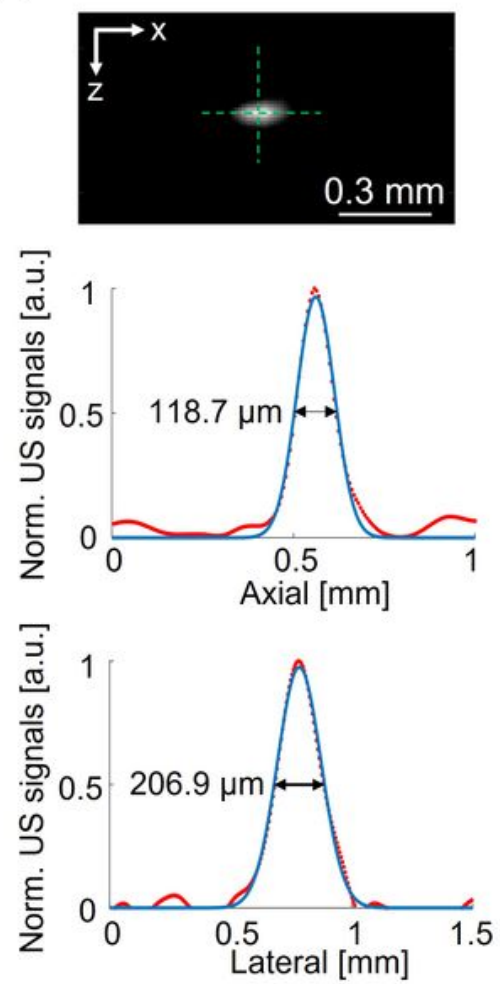

(e)

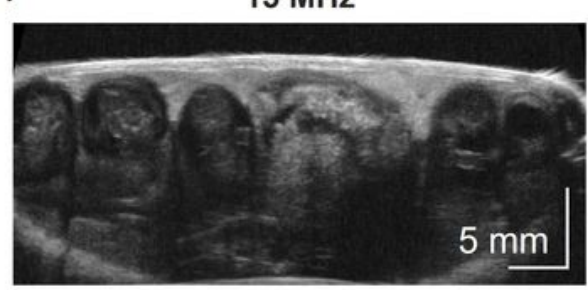

$25 \mathrm{MHz}$

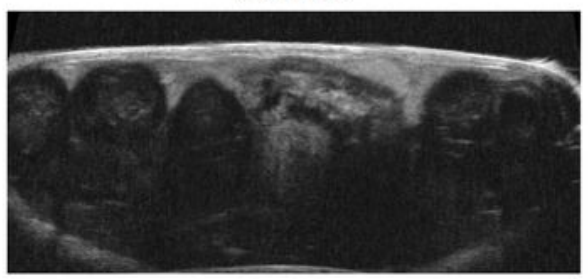

$35 \mathrm{MHz}$

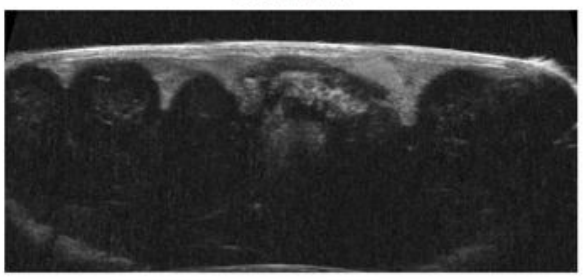

\section{Figure 1}

Integrated photoacoustic, ultrasound and angiographic tomography (PAUSAT). (a) Schematic of the PAUSAT system, showing the key acoustic, optical and mechanical components. (b) Imaging principles of the PA, US, and AA modes. In the PA mode, the pulsed laser light is delivered by the optical fiber bundle 
and the resultant acoustic waves are detected by the linear transducer array; In the US mode, the highfrequency inner-element of the focused wobbler transducer transmits the acoustic waves and detects the reflected echo signals; In the AA mode, the low-frequency outer-element of the wobbler transmits a strong acoustic pulse to burst the flowing microbubbles in the blood vessels, and the high-frequency innerelements detects the bursting signals from the bubbles. (c) Spatial resolution measurement of the PA mode. (d) Spatial resolution measurement of the US mode. (e) Comparison of the penetration depth of the US mode at three different frequencies.

(a)

PA

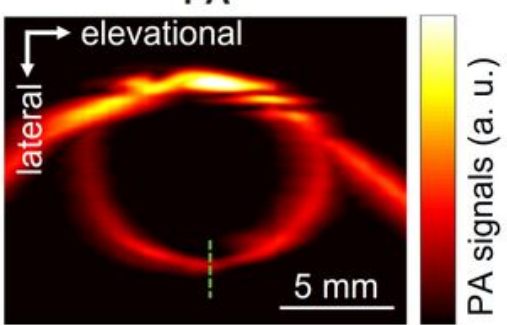

(b)

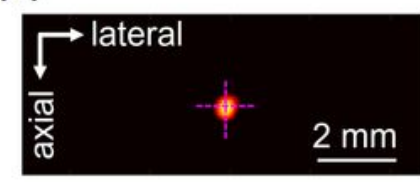

US

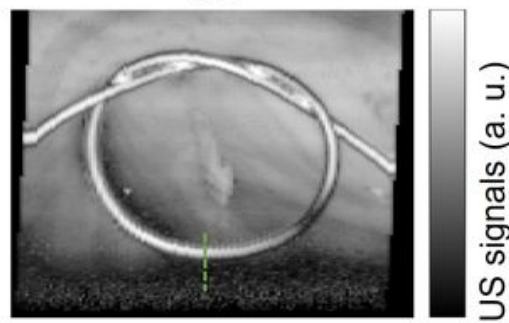

AA

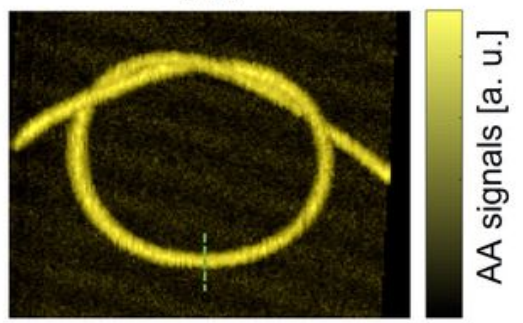

(c)
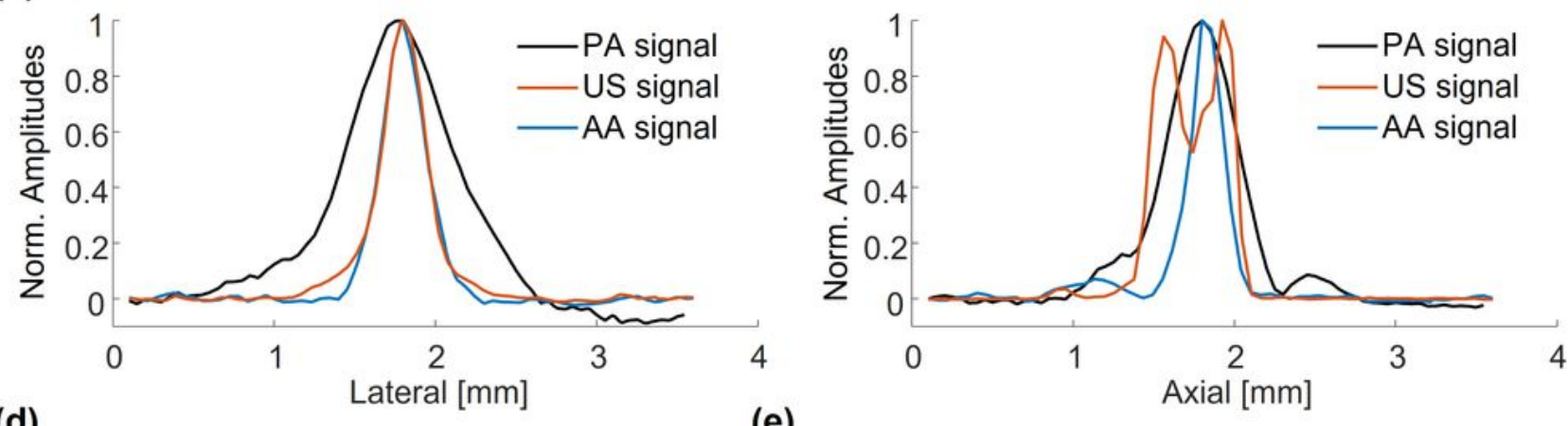

(d)
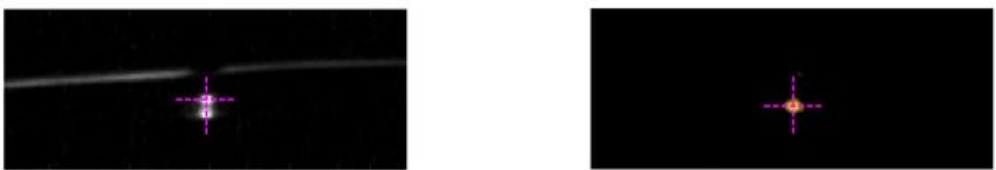

(e)
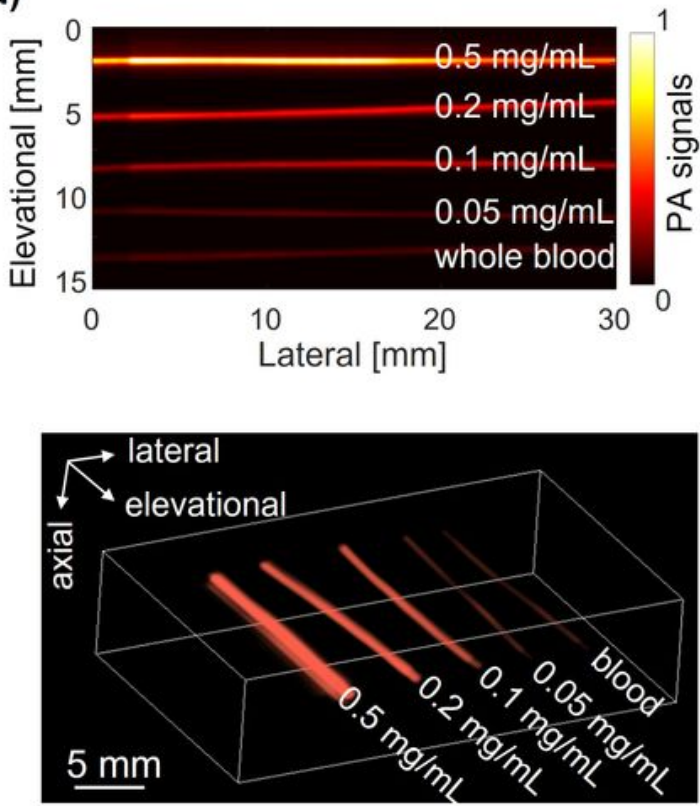

(f)
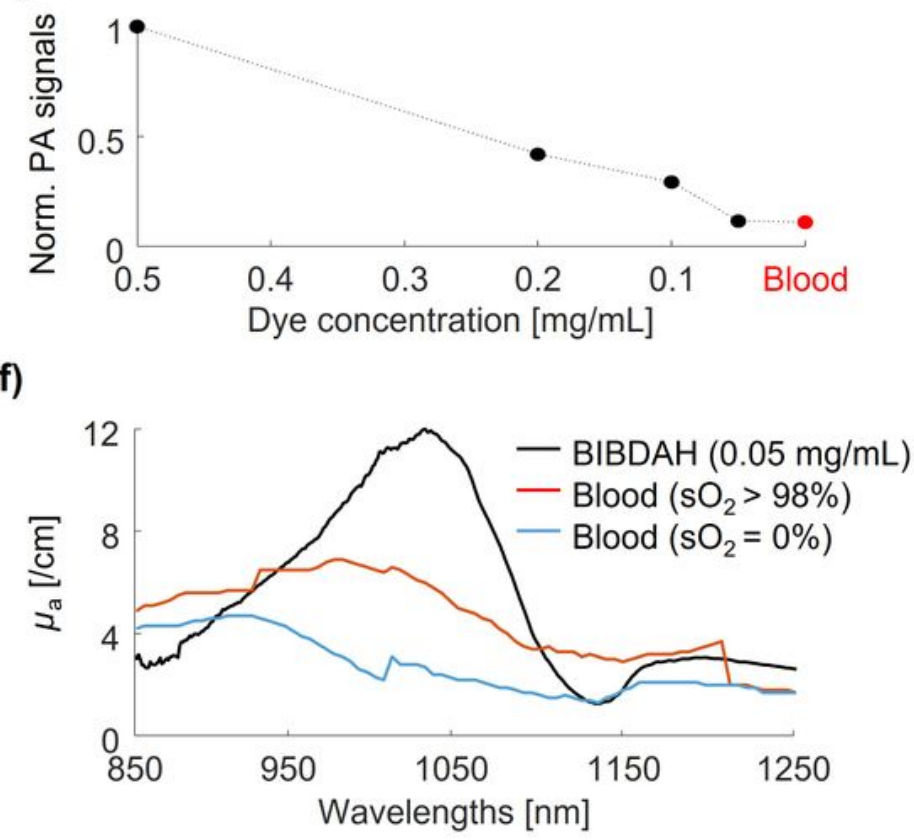
Figure 2

PAUSAT system validation on phantoms. (a) Images of a tube knot filled with BIBDAH, water, and microbubbles with PA, US, and AA, respectively. (b) Cross-sectional images of the tube along the greendotted lines in (a). (c) Lateral and axial signal profiles along the magenta dotted lines in (b). (d) Top-view projection PA image and 3D rendering of tubes filled with blood and BIBDAH dye at different concentrations. (e) Quantitative PA signals from blood and dyes. (f) Optical absorption spectra of BIBDAH at $50 \mu \mathrm{g} / \mathrm{ml}$, oxygenated and deoxygenated blood. 
(a)

(b)
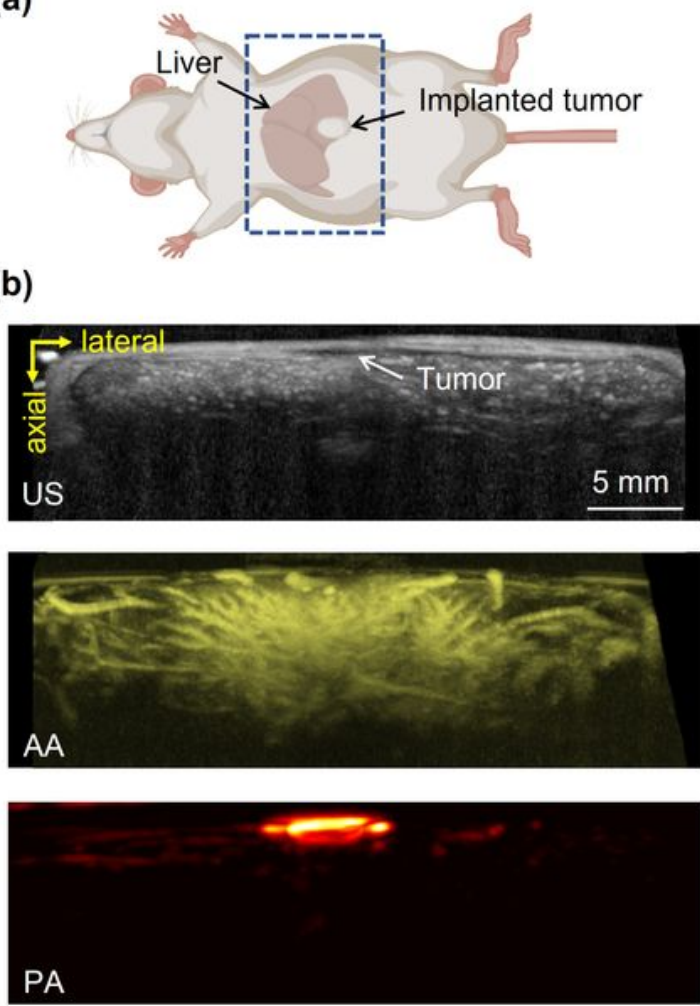

(d)

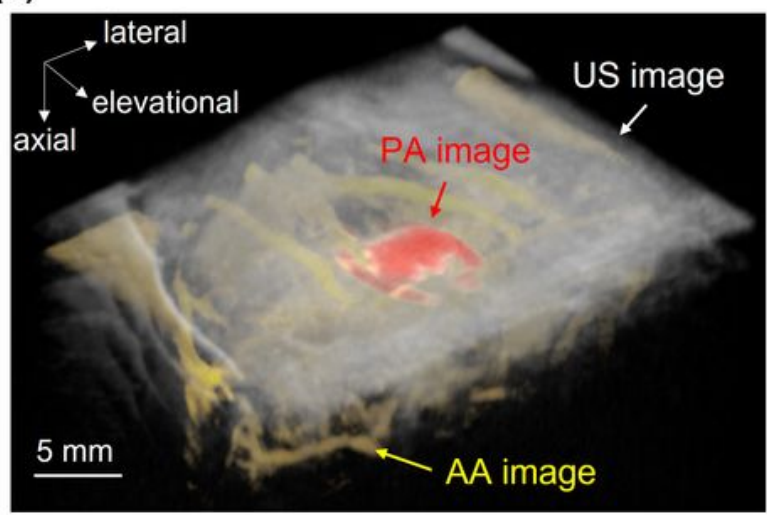

(c)
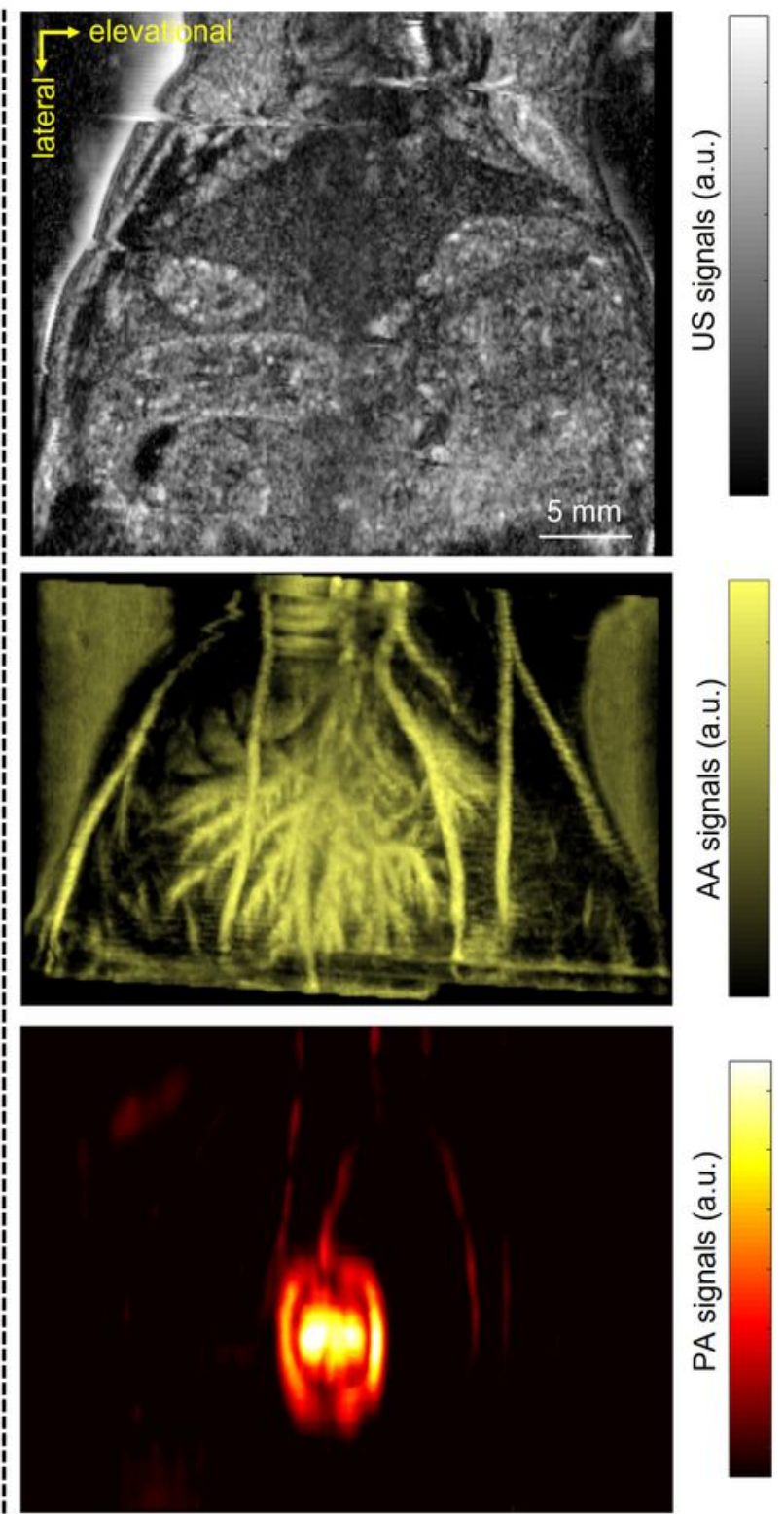

(e)

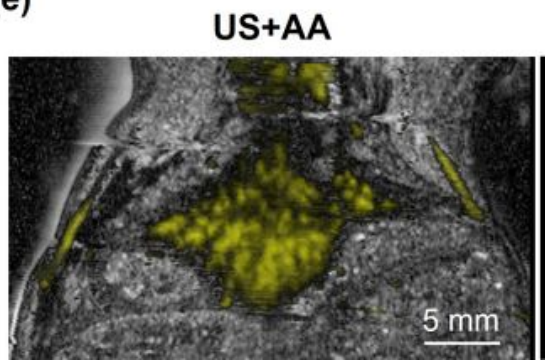

US+PA

US+AA+PA
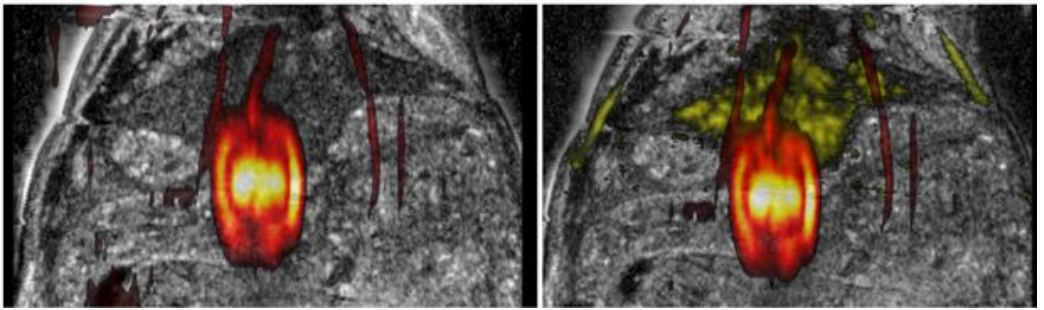

\section{Figure 3}

PAUSAT system validation on small animals. (a) The implantation position of the BIBDAH dye mixed Matrigel tumor. (b) Axial cross-sectional images of the mouse liver region by the PA, US, and AA mode. (c) Coronal images of the liver region by the PA, US, and AA mode. US shows one slice where the liver was located while AA and PA show the maximum amplitude projections. (d) Three-dimensional rendering of 
the superimposed PA (in hot color), US (in gray color), and AA (in yellow color) images. (e) Superimposed images of different imaging modes showing the relative positions of the targets.

(a)

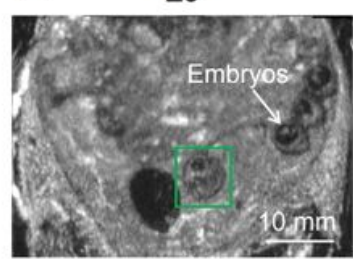

(b)

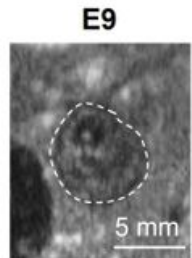

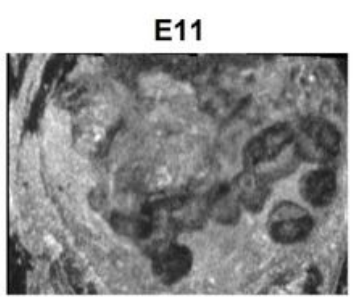
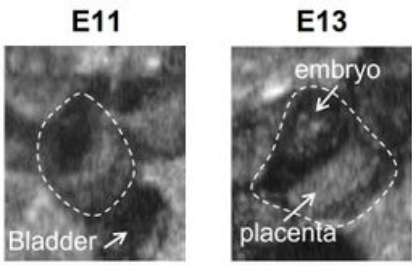

E11

E9

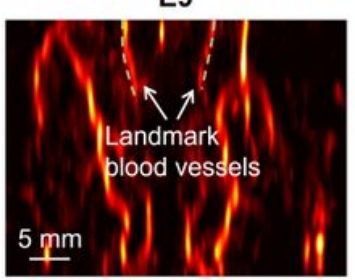

(e)

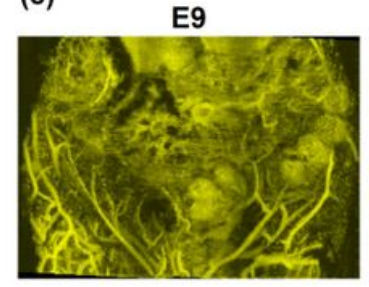

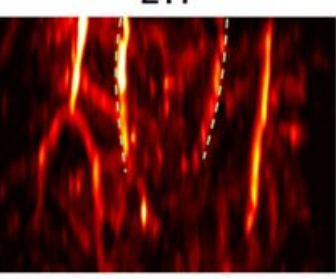

E13

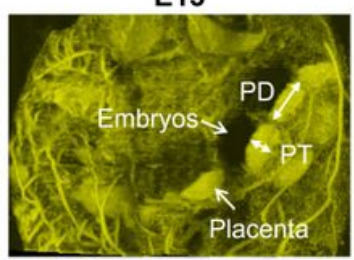

E13

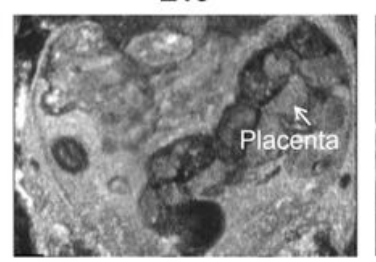

E15

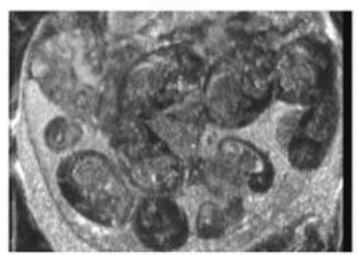

(c)
E15

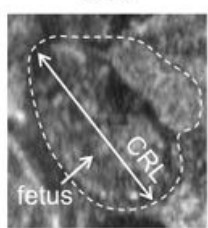

E17
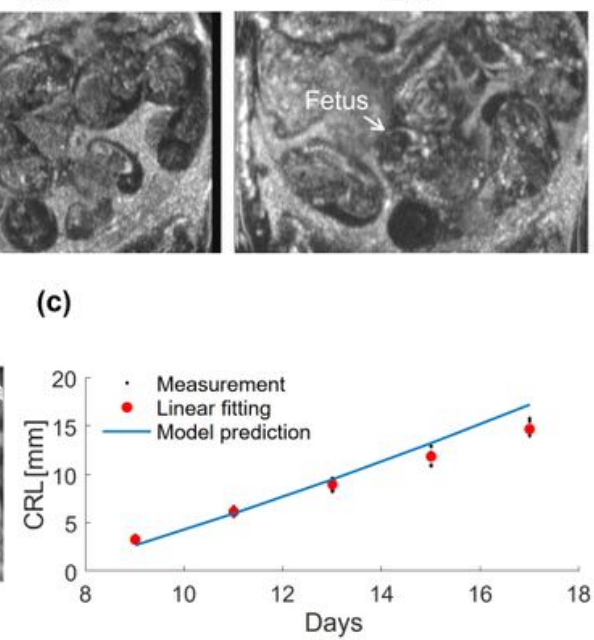

E17
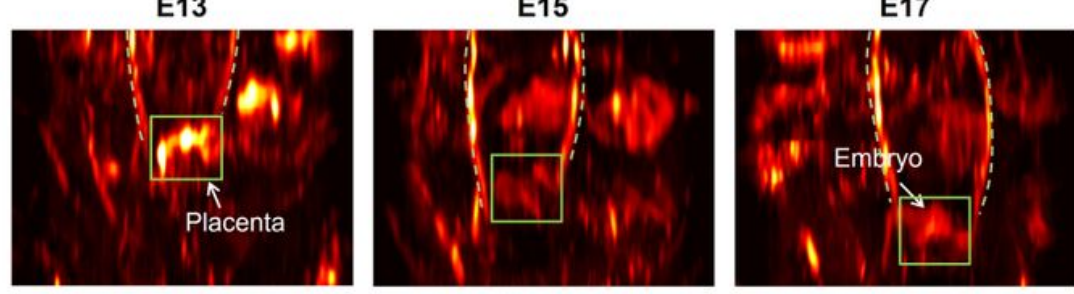

(f)

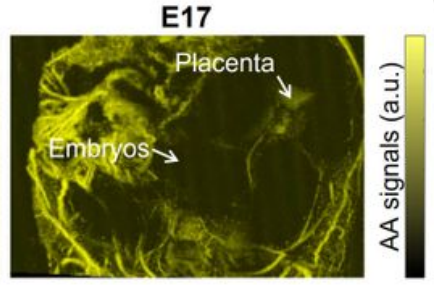

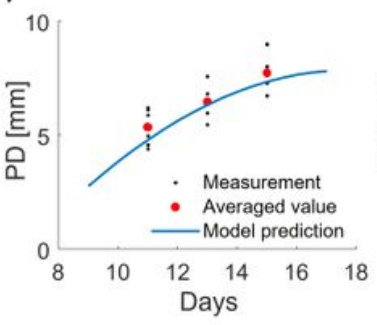

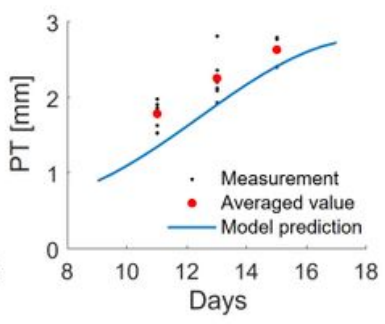

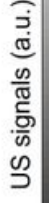
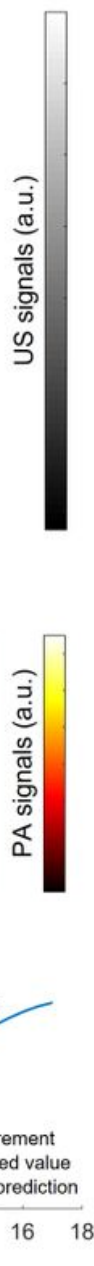

Figure 4

(a) Longitudinal US images of a pregnant mouse at E9, E11, E13, E15 and E17. (b) Close-up images of a single embryo from E9 to E17. (c) Embryo's crown-rump length measured from the US images (black dots), the linear fitting (red dots), and the model prediction (blue curve). (d) Longitudinal PA images of the pregnant mouse. (e) Longitudinal AA images of the maternal vasculature and placentas. (f) Placental diameter (PD) and placental thickness (PT) measurements from the US and AA images for individual embryos (black dots), the averaged values (red dots), and the model prediction (blue curves). 
(a)
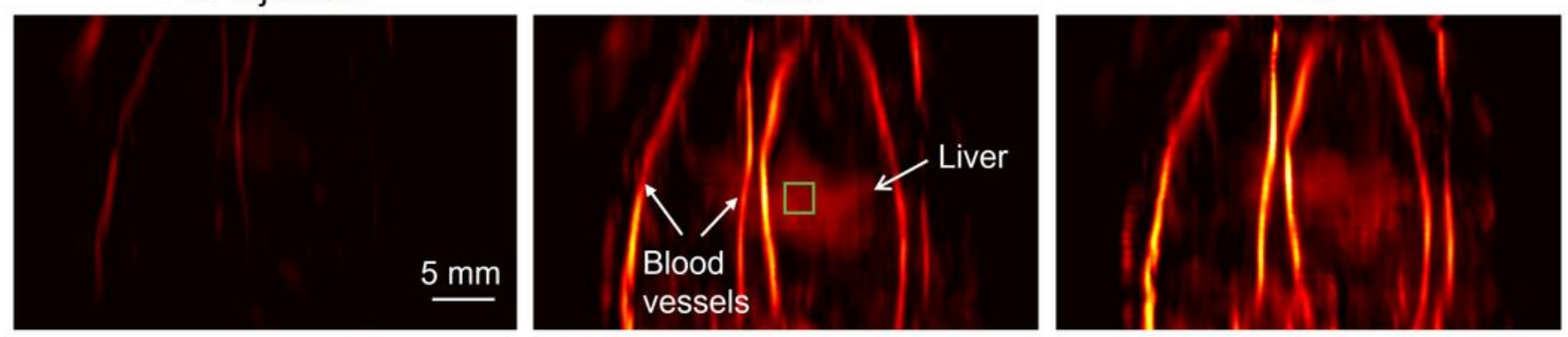

$2 \mathrm{~h}$

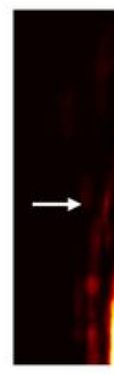

(b)
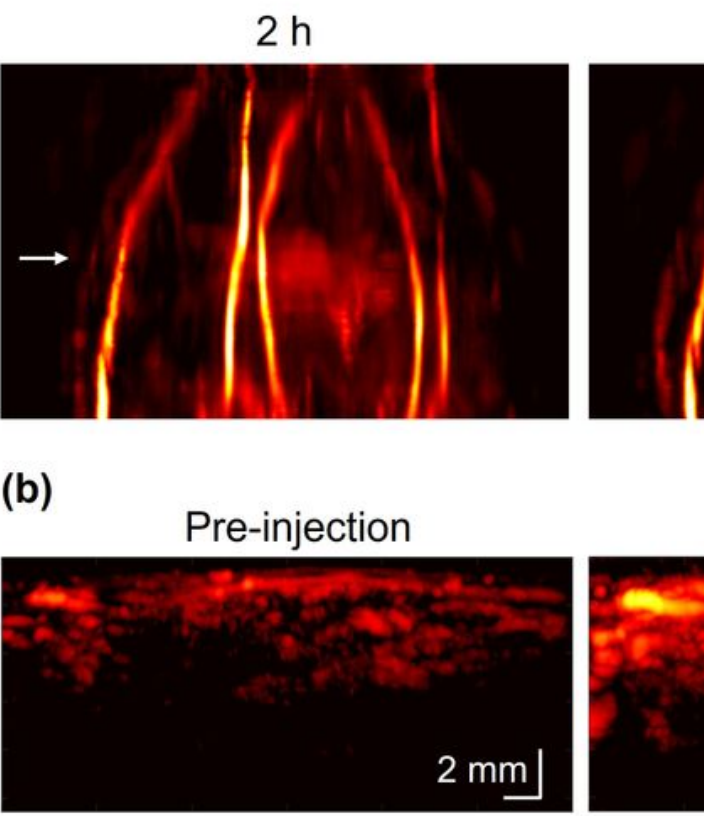

$3 \mathrm{~h}$

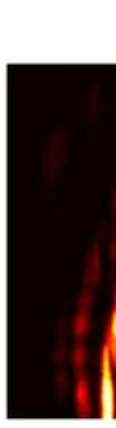

$4 \mathrm{~h}$

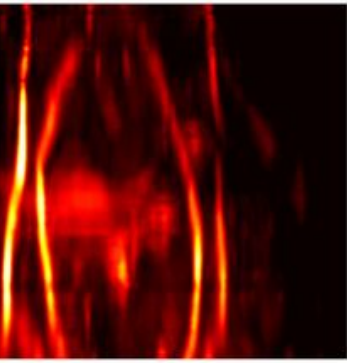

2h post-injection

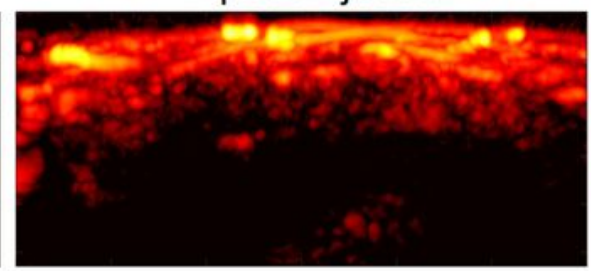

(c)

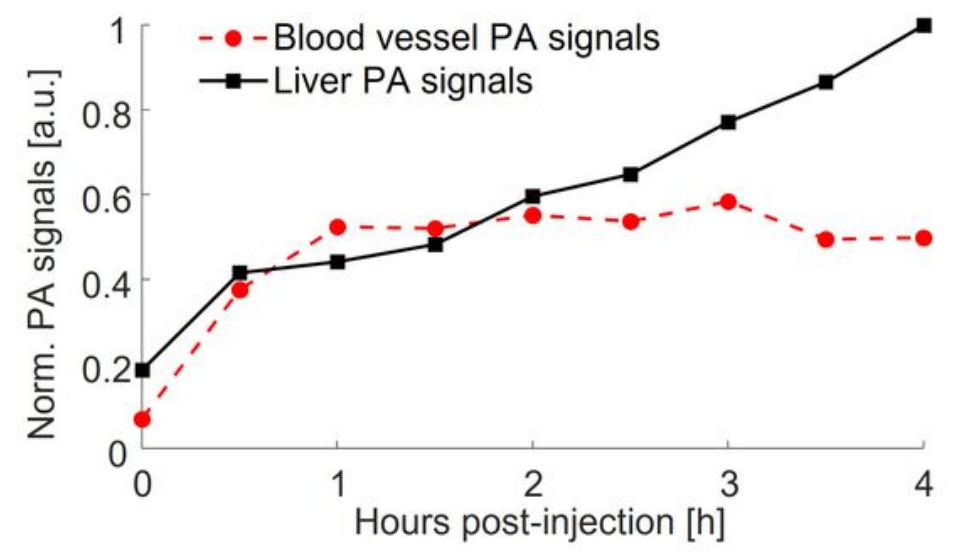

(d)

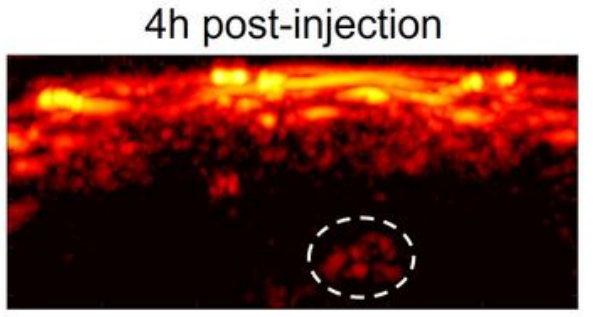

\section{Figure 5}

Tracking the biodistribution of an NIR-II dye. (a) PA images of the mouse liver region before and after the injection of an NIR-II dye BIBDAH. (b) Axial cross-sectional images of the liver region indicated by the white arrow in (a). (c) PA signal dynamics in the major blood vessels and in the liver region as indicated by the green square in (a). (d) 3D rendering of the superimposed US (shown in gray) and PA (shown in color) image of the mouse 4 hours post-injection. 
(a)

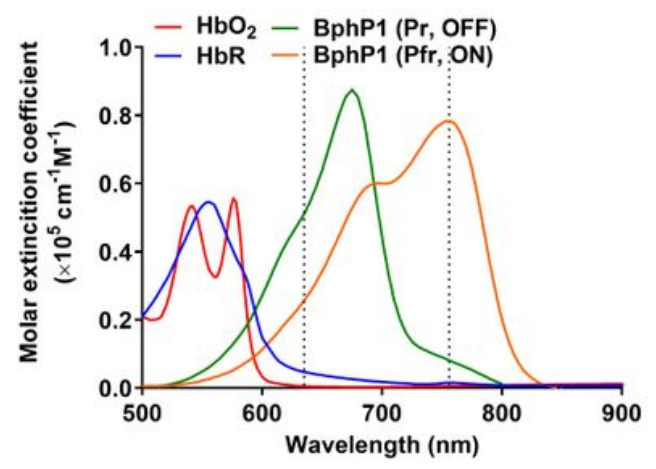

(d)

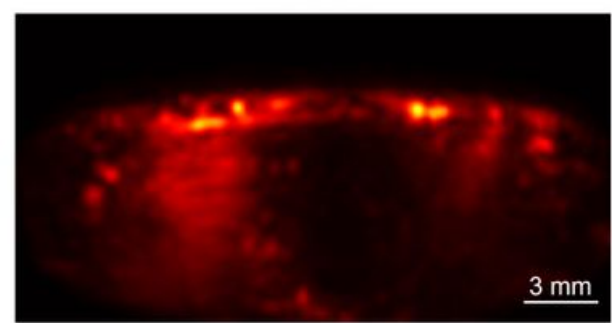

PA signals (a.u.)

(f)

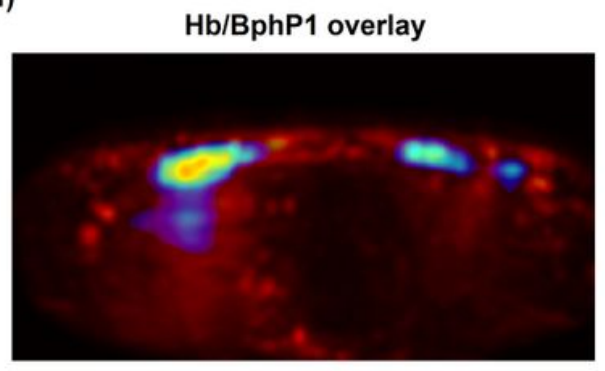

(b)

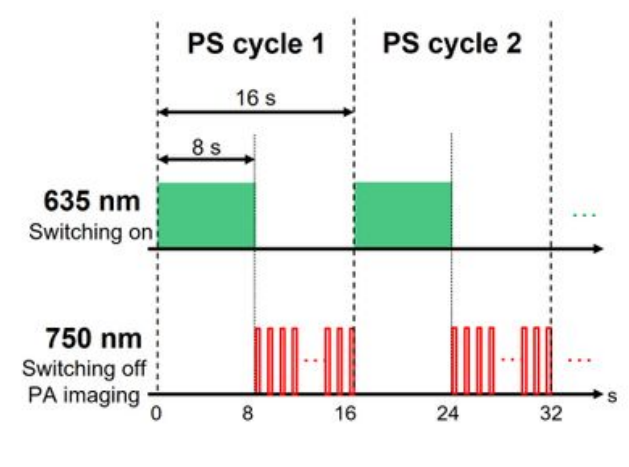

(e)

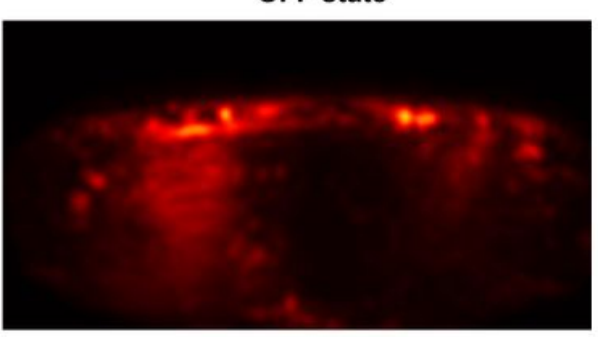

OFF state

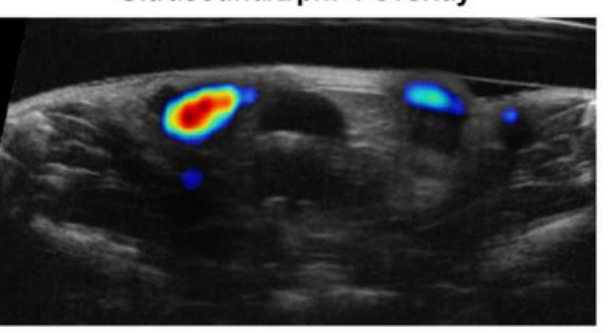

(c)
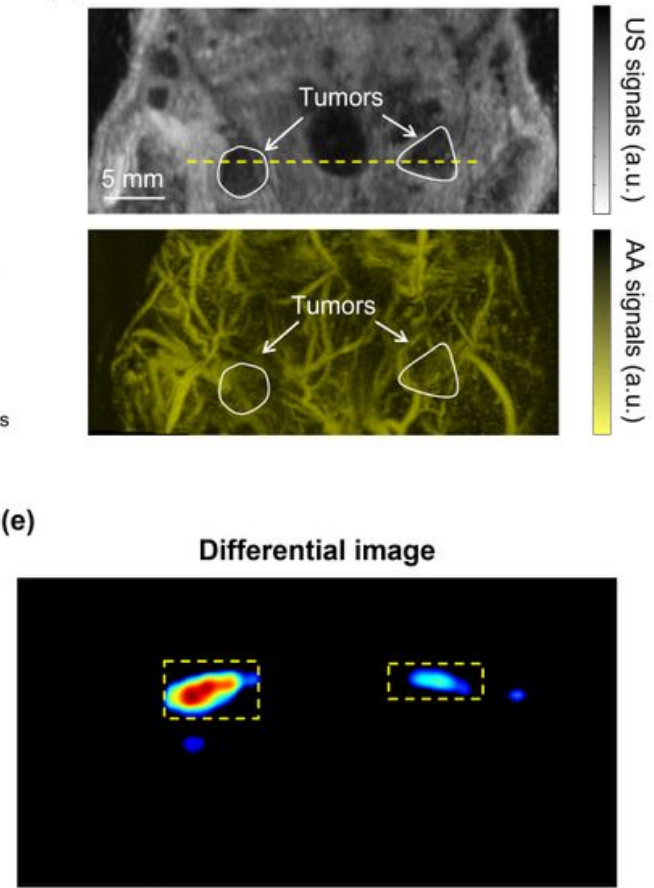

Bphp1 signals (a.u.)

(g)

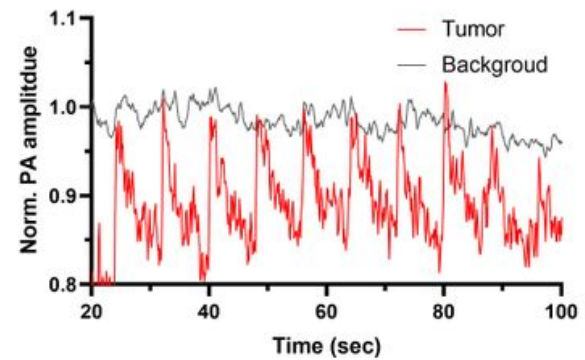

Figure 6

Detecting reversibly photoswitching tumor in vivo. (a) Molecular extinction coefficients of oxygenated hemoglobin ( $\mathrm{HbO} 2$, red curve), deoxygenated hemoglobin ( $\mathrm{Hb}$, blue curve), ON state Bphp1 (orange curve) and OFF state Bphp1 (green curve) from $500 \mathrm{~nm}$ to $900 \mathrm{~nm}$. (b) PA laser excitation sequence used for photoacoustic imaging and photoswitching Bphp1. The PA images were acquired at $750 \mathrm{~nm}$. (c) Coronalview US and AA images of the mouse tumor region. The circles indicate the positions of implanted tumors. (d) Axial cross-sectional PA images at the ON and OFF state of the Bphp1 tumor. The position of the cross-section is indicated in the US image in (c). (e) Differential PA image between the ON and OFF state, showing the tumor only. (f) The differential image overlayed with PA and US images. (g) PA signal changes from tumor and blood over multiple photoswitching cycles.

\section{Supplementary Files}

This is a list of supplementary files associated with this preprint. Click to download. 
- Supplementary1Wobberscanning.mp4

- Supplementary2AnimalValidation.mp4

- Supplementary3PregnantMouseUSimages.mp4

- Supplementary4PregnantMousePAimages.mp4

- Supplementary5PregnantMouseAAimages.mp4

- Supplementary6PregnantMouseAA.mp4 